\title{
Analysis of the circumstellar environment of the $B[e]$ star HD 45677 (FS Canis Majoris) ${ }^{\star}, \star \star$
}

\author{
G. Muratorio ${ }^{1}$, C. Rossi ${ }^{2}$, and M. Friedjung ${ }^{3}$ \\ ${ }^{1}$ Laboratoire d'Astrophysique de Marseille, 2 Place le Verrier, 13248 Marseille Cedex 04, France \\ e-mail: gerard.muratorio@oamp.fr \\ 2 Dipartimento di Fisica, Università "La Sapienza", Piazzzale A. Moro 2 00185, Roma, Italy \\ e-mail: corinne.rossi@roma1.infn.it \\ ${ }^{3}$ Institut d'Astrophysique, 98 bis Boulevard Arago, 75014 Paris, France \\ e-mail: fried@iap.fr
}

Received 29 April 2005 / Accepted 29 December 2005

ABSTRACT

Aims. We studied the circumstellar environment of the B[e] star HD 45677 through the analysis of the emission lines from ionized metals. Methods. We used the statistical approach of the self absorption curve method (SAC) to derive physical parameters of the line emitting region. Results. The Fe II and Cr II double-peaked emission line structure is explained by the presence of a thin absorption component red shifted by $\sim 3 \mathrm{~km} \mathrm{~s}^{-1}$. This absorption component can be interpreted geometricaly as being due to infalling material perpendicularly to the disk seen nearly pole-on, as indicated by the emission line structure. The $\mathrm{Cr}$ II and Fe II emission lines have a complex structure with two (narrow and broad) components, of 45 and $180 \mathrm{~km} \mathrm{~s}^{-1} F W H M$ for the permitted lines and 25 and $100 \mathrm{~km} \mathrm{~s}^{-1} F W H M$ for the forbidden ones, respectively. From our best data set of 1999, we obtained a Boltzmann-type population law whose excitation temperature is $3900_{-600}^{+900} \mathrm{~K}$ and $3150_{-300}^{+350} \mathrm{~K}$ for the narrow component lower and upper levels respectively. We obtained an excitation temperature of $3400_{-300}^{+350} \mathrm{~K}$ for the broad component upper levels. The forbidden lines are found to be formed in the outer regions with higher excitation temperatures of $10500 \pm 1000 \mathrm{~K}$ and $8000 \pm 1500 \mathrm{~K}$ for the narrow and broad components respectively in 1999. Our results are consistent with line formation in a rotating disk, around a young star. In the framework of a very simplified geometrical model, we argue that the narrow components are principaly emitted by an optically thin disk seen nearly pole-on, in a region whose minimum radius is estimated to be $4 \times 10^{12} \mathrm{~cm}$, while the broad ones are formed in a disk-linked wind.

Key words. line: formation - methods: data analysis - stars: emission-line, Be - stars: individual: HD 45677 (FS Canis Majoris)

\section{Introduction}

HD 45677 (FS CMa) is generally recognized as an early type star, usually classified as $\mathrm{B}[\mathrm{e}]$, or more precisely as showing the $\mathrm{B}[\mathrm{e}]$ phenomenon, even if it is difficult to determine its exact evolutionary stage (de Winter et al. 1997; Lamers et al. 1998).

The presence of a disk, and even more of a bipolar environment, was inferred by polarization measurements (Coyne \& Vrba 1976; Schulte-Ladbeck et al. 1992) and by UV spectroscopic measurements of accreting gas (Grady et al. 1993). These last authors agreed on the presence, around the star, of an actively accreting circumstellar, protoplanetary disk, and the presence of low-velocity absorption profiles in Fe II lines, among other species, while optical spectroscopic measurements of strong absorption cores of a few lines are commented on by de Winter et al. (1997).

\footnotetext{
* Based on observations obtained at the Haute Provence Observatory.

$\star \star$ Tables 1-3 and Fig. 3 are only available in electronic form at http://www.edpsciences.org
}

In the optical HD 45677 is characterised by a rich emission line spectrum, including the Balmer lines of hydrogen, and both permitted and forbidden lines of neutral and ionized metals (Mg I, Mg II, Na I, Cr II, Si II, Mn II, Ti II, [Ni II], [S II] and especially $\mathrm{Fe}$ II and [Fe II]).

Israelian et al. (1996) found a surface gravity of $\log g=$ $3.85 \pm 0.05$ (luminosity class $\mathrm{V}$ ) from the photospheric wings of $\mathrm{H} \gamma$ and $\mathrm{H} \delta$, while examination of the $\mathrm{He}$ I absorption lines suggested a spectral class of B2 $\left(T_{\text {eff }}=22000 \pm 1500 \mathrm{~K}\right)$. The effective temperature was less certain than the surface gravity, due to a circumstellar contribution to the $\mathrm{He}$ I lines. Israelian et al. (1996) also found that the photospheric Si II 4128 and $4130 \AA$ lines were not blended, and could be only fitted by models with a projected rotational velocity $v \sin i$ of less than $70 \mathrm{~km} \mathrm{~s}^{-1}$.

Cidale et al. (2001) used the method of Barbier, Chalonge \& Divan, based on the magnitude and position of the Balmer photospheric (not circumstellar) discontinuity. They obtained a spectral type of B2IV-V, corresponding to $T_{\text {eff }}=21500 \pm 300 \mathrm{~K}$ 


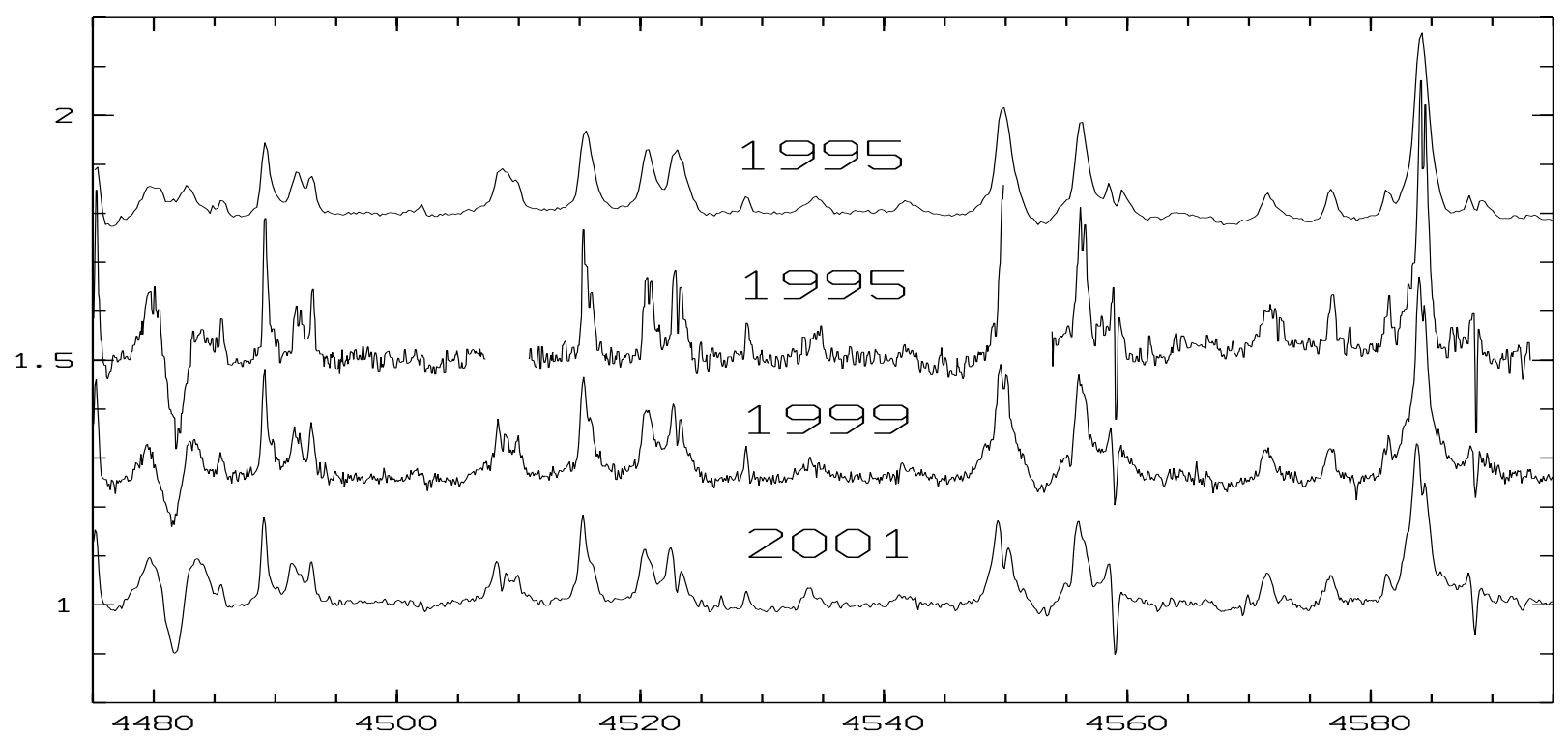

Fig. 1. The spectrum of HD 45677 during 1995-2001. The spectrograms, normalized to the continuum and vertically shifted, are, from top to bottom, of $14 / 01 / 1995(R=7000), 19 / 10 / 1995(R=36000), 2 / 2 / 1999(R=15000)$ and $5 / 12 / 2001(R=15000)$. All the spectra were obtained at OHP with AURELIE, except the October 1995 one which is from the Russian Special Astrophysical Observatory. On the higher resolution spectrograms the deep Cr II absorptions at 4558 and $4588 \AA$ A show up clearly.

and $\log g=3.89$, in agreement with the previous determinations.

De Winter \& van den Ancker (1997) studied the behaviour of HD 45677 during the last 25 years, and concluded that the large photometric variations of the star are best explained by a variable obscuration from circumstellar grains. They also derived a stellar instrinsic visual-ultraviolet energy distribution close to that of a B2 V star, with an infrared excess due to the dust emission. The recent spectral variations were discussed by Israelian et al. (1996) and Israelian \& Musaev (1997).

In the present study, we analyse the emission line spectrum in order to get an insight into the physical processes of line excitation and geometrical conditions of their forming regions. It is clearly impossible at the present stage to calculate a detailed synthetic spectrum for this badly known object, so in order to analyse the spectrum, we use the statistical approach offered by the Self-Absorption Curve method (Friedjung \& Muratorio 1987; Muratorio \& Friedjung 1988; Baratta et al. 1998; Kotnik-Karuza et al. 2002).

\section{Observations and reductions}

The spectra of HD 45677 were obtained in various contiguous domains in the course of different observing runs at the Haute Provence Observatory (OHP) by means of the Aurelie spectrograph attached to the Coude focus of the $1.52 \mathrm{~m}$ telescope.

Until 1999 Aurelie used as receiver a Thomson TH 7832 photocell "Double Barette", and since 2000 a new CCD/EEV which improved the instrument performances (see http:// www . obs-hp. fr/www/guide/aurelie/annonce.html).

We obtained many spectra in selected regions; A higher resolution was used in 1999 and 2001-2002 in order to characterise better the profile of the metallic spectral lines. A detailed $\log$ of the observations is given in Table 1 (online material).
The 1995 observations were used in 1996 for the analysis of the He I lines 4387, 4471, 5876 and $4438 \AA$ (Israelian et al. 1996).

The spectra were reduced at the Marseille Observatory using procedures runing in the MIDAS environment.

After the files were calibrated in wavelength, and reduced to the heliocentric standard of rest, we identified the spectral features present in all our spectra. No large variations are present in the metallic emission lines within our observing time target (see Fig. 1). Higher resolution spectra of HD 45677 were obtained from October 14, 19 and 20 in 1995 by Musaev with the Coudé Echelle Spectrometer at the 1-m telescope of the Special Astrophysical Observatory of the Russian Academy of Sciences (Israelian \& Musaev 1997), and kindly provided for us by Garik Israelian.

For the purpose of our analysis of the emission lines we need the absolute values of the fluxes. To do this, we have normalized the individual spectra to the local continuum level and, following de Winter et al. (1997), we multiplied the line fluxes by the energy distribution of the Kurucz $(1979,1993)$ model of $T_{\text {eff }}=22500 \mathrm{~K}$ and $\log g=4.0$ with solar abundance, normalized to the visual magnitude of the star, $V_{0}=6.6$. The results are not dependent on the model choosen in the limit of the precision of the determination of the star's type by various authors (see Introduction). We adopted the same continuum distribution for the three studied epochs.

\subsection{Line identification}

Extensive line identification lists of HD 45677 are available from the literature (Swings 1973; Andrillat et al. 1997; de Winter et al. 1997). However, they generally refer either to lower resolution spectra and/or to limited spectral ranges. We therefore prepared a new line list, based on our observations, 

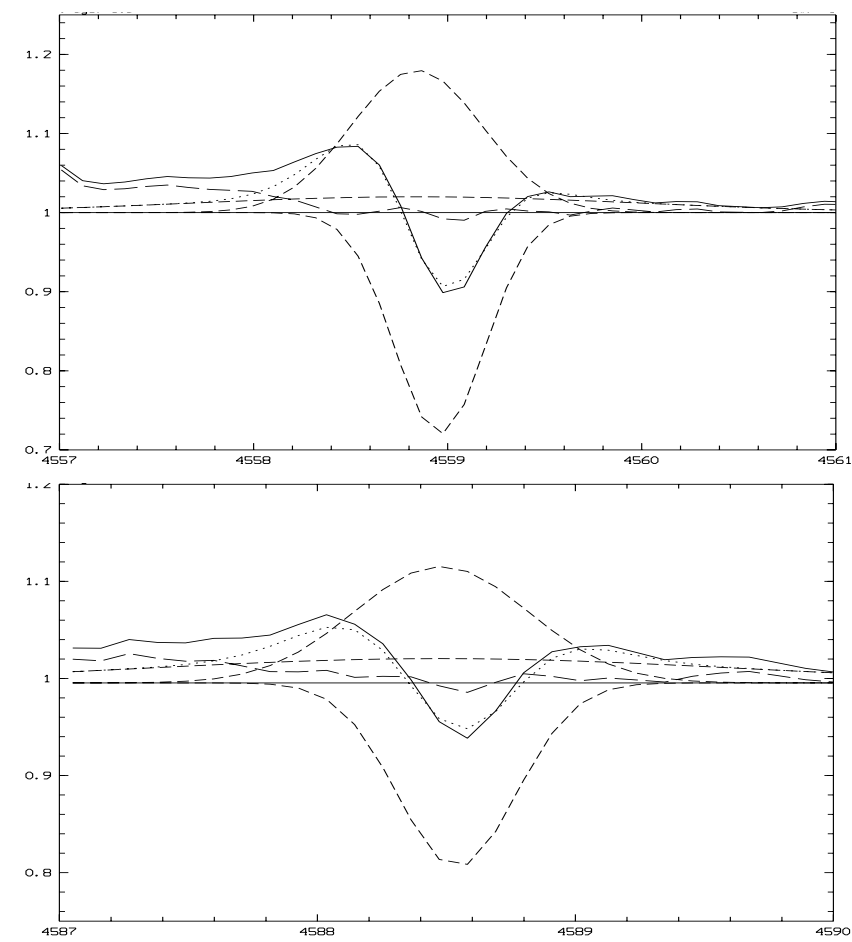

Fig. 2. The two most intense lines of multiplet 44 of $\mathrm{Cr}$ II observed the $5 / 12 / 2001$, showing the central absorption well below the continuum and the three Gaussian components' fit. Each line profile (solid line) is fitted (dotted line) by three Gaussian components (dashed line). The residual (long dashed line) appears around the continuum (straight line). Notice the rising of the residual towards shorter wavelengths which is in both cases due to the presence of a strong Fe II line.

which is presented in Table 2 (online material). Emission and absorption components identified in different species are summarized in Table 3 (online material).

\subsection{Line components}

Figure 1 shows that the lines of the most numerous species, namely $\mathrm{Cr}$ II, $\mathrm{Fe}$ II and [Fe II ], did not significantly vary during 1995-2002. This will be best illustrated at the end of this section.

As shown in Fig. 1, and, more in detail, in Figs. 2-5, in the higher resolution spectra of HD 45677, the strongest permitted lines present a double peaked emission. This feature has been in previous papers ascribed to emission from a rotating equatorial disk (de Winter et al. 1997). But they can be as well attributed to absorption in the emitting medium or in a circumstellar shell according to Grady et al. (1993) from their ultraviolet observations.

Israelian \& Musaev (1997) discussed the narrow absorption components observed in $\mathrm{Mg}$ II $\lambda 4481 \AA$ and in the hydrogen lines in the framework of both infalling material with a patchy structure, and outfall-wind. They also noticed the strong variations of the $\mathrm{Mg}$ II line from pure absorption to P Cygni profile on timescales of a few days.

Actually, in the higher resolution spectra the central dips of the strong Mg II $\lambda 4481 \AA$ and $\mathrm{Cr}$ II $\lambda 4558.6 \AA$ and $\lambda 4588.2 \AA$ go well below the continuum level (Figs. 1 and 2).

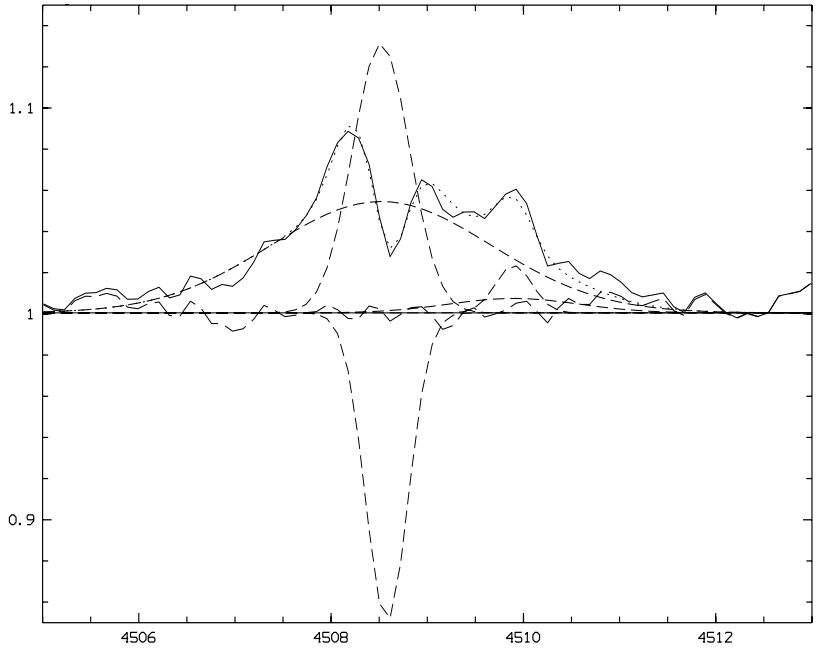

Fig. 4. Profiles and various components of the blend $4508.288 \AA$ (Fe II multiplet 38), 4509.610 $\AA$ ([Fe II] multiplet 6F) on the 2/2/1999 (see Fig. 2 for the explanation of the different curves). The different component $F W H M$ are given in the text.

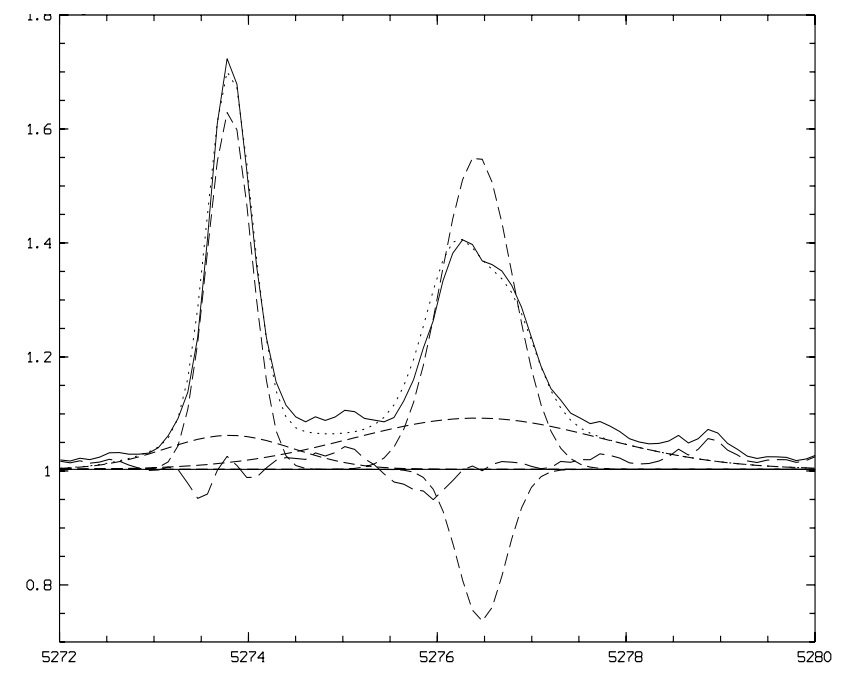

Fig. 5. Profiles and various components of $5273.38 \AA$ ([Fe II] multiplet $18 \mathrm{~F}$ ), 5276.002 $\AA$ (Fe II multiplet 49), and 5278.374 $\AA$ ([Fe II] multiplet 35F) on the 3/2/1999 (see Fig. 2 for the explanation of the different curves).The different component $F W H M$ are given in the text.

In our lower resolution 1995 spectra (top), the absorption is probably smoothed out, but is nevertheless present, as confirmed by the fitting procedure discussed below. The other emission lines are either sligthly flat-topped or present a dip at the red side of their maximum, again suggesting the presence of an unresolved central (or slightly red-shifted) absorption.

Indeed, the line shape is that typical of an emission line with a central absorption, rather than that of a rotating disk seen close to edge-on and we shall see in Sect. 4 that these profiles can be interpreted as originating in a nearly face-on seen disk.

At the same time, the strongest emission lines clearly present extended wings, that, however, are difficult to detect in many cases because of line blending.

In Fig. 2 we show in more detail the two most intense lines of $\mathrm{Cr}$ II multiplet 44 . The line profiles are well reproduced with 
Table 4. Flux (in $10^{-13} \mathrm{erg}^{2} \mathrm{sm}^{-1}$ ) and velocity (in $\mathrm{km} \mathrm{s}^{-1}$ ) variations of the Na I D1 and D2 components.

\begin{tabular}{lllllll}
\hline \hline & $\begin{array}{l}1995 \\
\text { flux }\end{array}$ & $\begin{array}{l}1995 \\
\text { vel }\end{array}$ & $\begin{array}{l}1999 \\
\text { flux }\end{array}$ & $\begin{array}{l}1999 \\
\text { vel }\end{array}$ & $\begin{array}{l}2002 \\
\text { flux }\end{array}$ & $\begin{array}{l}2002 \\
\text { vel }\end{array}$ \\
\hline Na I D1 a & -0.88 & 31 & -1.85 & 24 & -2.60 & 24 \\
Na I D1 e & 2.77 & 48 & 4.84 & 4 & 2.86 & 9 \\
Na I D2 a & -1.26 & 31 & -2.05 & 22 & -2.55 & 22 \\
Na I D2 e & 3.34 & 48 & 5.24 & 5 & 2.88 & 6 \\
\hline
\end{tabular}

a multiple Gaussian fit, with two components in emission with a $F W H M$ of 45 and $180 \mathrm{~km} \mathrm{~s}^{-1} F W H M$ respectively, having the same central wavelength, and one absorption with a $F W H M$ of 25-30 $\mathrm{km} \mathrm{s}^{-1}$, slightly displaced to the red. (See the infalling material model discussed by Israelian and Musaev.)

Strong absorption components have also been observed in the $\mathrm{Na}$ I $\mathrm{D}$ doublet since a long time, and were discussed by de Winter et al. (1997). In our spectra the $\mathrm{Na}$ I $\mathrm{D}$ doublet shown in Fig. 3 underwent dramatic variation in both intensity and position of the emission components, as well as in the intensity of the central absorption (its position is stable within the fitting precision). This behaviour is not unexpected because Israelian et al. (1996) report variability for these lines within 2-3 days. Due to the intense absorption, the narrow emission component, if present, cannot be found by the fitting procedure. The velocity and fluxes of absorption and broad emission components are given for the three epochs in Table 4.

As for the Fe II lines, a central absorption is clearly present in the line profiles at $\lambda 4508.288 \AA$ in Figs. 1 and 4 and at $\lambda 4520.224 \AA, 4549.474 \AA$ and $4583.837 \AA$ in Fig. 1 . The absorptions, like the $\mathrm{Cr}$ II ones, are red shifted an average of $3 \mathrm{~km} \mathrm{~s}^{-1}$ with respect to the central wavelength of the emission; the shift is the same for all the observing runs (Table 5).

The same set of components than for $\mathrm{Cr}$ II lines with similar parameters is present in the Fe II lines ( $F W H M$ of 45 and $180 \mathrm{~km} \mathrm{~s}^{-1}$ ), while the [Fe II] display narrow and broad emissions with smaller $F W H M$ of 25 and $100 \mathrm{~km} \mathrm{~s}^{-1}$, respectively (see Fig. 4 for highly blended lines and Fig. 5 for more separated ones).

As the FWHM of the Fe II absorption components FWHM are similar to the $\mathrm{Cr}$ II ones $\left(\simeq 30 \mathrm{~km} \mathrm{~s}^{-1}\right)$, these components probably originate from the same region.

We postulate that in HD45677 the double-peaked Fe II line structure reported in the literature is in fact due to the same type of absorption and originates from material in the star's line of sight. Their redward displacement can be interpreted as due to the infall of the absorbing material. This result is in agreement with the observations and the conclusions of Grady et al. (1993) and Israelian \& Musaev (1997).

The two emission components clearly originate from regions of different dynamical behaviour, a possible model of the environment of FS CMa will be given in Sect. 4 .

Based on the above results, we have fitted all, strong and weak, metallic permitted lines identified in our spectra with three Gaussian line components, central absorption narrow emission and broad emission. The forbidden lines were fitted with two emission components. The same central wavelength
Table 5. Mean radial velocities (in $\mathrm{km} \mathrm{s}^{-1}$ ) of the most numerous species, absorption and emission of $\mathrm{Cr}$ II, Fe II, and [Fe II] emission. The number of lines is given in parentheses.

\begin{tabular}{llll}
\hline \hline & 1995 & 1999 & 2001 \\
\hline Cr II a & $25.4 \pm 1.3(10)$ & $25.4 \pm 1.4(11)$ & $21.8 \pm 0.8(4)$ \\
Cr II e & $23.6 \pm 1.3(11)$ & $23.1 \pm 1.2(11)$ & $18.6 \pm 2.0(4)$ \\
Fe II a & $29.4 \pm 0.7(58)$ & $28.2 \pm 0.6(59)$ & $24.2 \pm 0.6(35)$ \\
Fe II e & $25.8 \pm 0.5(69)$ & $24.5 \pm 0.4(64)$ & $21.6 \pm 0.5(47)$ \\
{$[$ Fe II] } & $21.5 \pm 0.5(85)$ & $22.5 \pm 0.4(77)$ & $21.4 \pm 0.3(32)$ \\
\hline
\end{tabular}

was assumed for the two emission components. The derived line fluxes, FWHMs and central radial velocities are given in Table 2 (online material). For the line fluxes we estimated from the fitting procedure an error from $15 \%$ for the strongest lines to $50 \%$ for the weakest lines.

The mean heliocentric radial velocity of the narrow emission and central absorption components of $\mathrm{Cr}$ II, $\mathrm{Fe}$ II and [Fe II] in the three epochs are given in Table 5.

We compared the fluxes of the narrow emission components of Fe II and [Fe II] lines in 1995 and in 2001-2002 with those of 1999.

Between 1995 and 2001-2002 the fluxes of the permitted lines are the same within the measurements errors, except for the weaker lines which are fainter in 1995. In December 2001January 2002 the forbidden lines have weakened by somewhat less than $30 \%$ with respect to 1995 and 1999 . As for the broad components, their larger measurement errors only allow to put an upper limit of $30 \%$ to their variation during our observing period.

In the following we shall analyse the broad and narrow emission components of these species, with special regard to the February 1999 data which cover a wider spectral range.

\section{Analysis of the emission line fluxes}

The spectrum of HD 45677 is rich in emission lines (see online material Table 3), whose strength can be used to derive information about the physical parameters and geometry of the circumstellar region. Here we analyse the intensity of the permitted and forbidden ionized iron emission lines and permitted ionized chromium emission lines in a statistical way, the Self-Absorption-Curve Method (SAC), following the procedure described by Friedjung \& Muratorio (1987), and used among others by Muratorio et al. (1992), Viotti et al. (1999), Viotti et al. (2000), Kotnik-Karuza et al. (2002) and van den Ancker et al. (2004). Details on the SAC method are given in the SAC Manual (Baratta et al. 1998, available at www.rm.iasf.cnr.it/ftp/uvspace/).

The Self absorption Curve Method has been discussed by Kastner (1999), in the framework of the escape probability formalism of radiative transfer. He found, with such a formalism, that for a static source having negligible differential velocities, the method is valid up to optical thicknesses of about 10 . The self absorption curve we use is very similar to that found from Kastner's approach. As we shall see, the use of a static model can be justified for one of the emission line components, while 
most of the Fe II emission lines examined, do not have an optical thickness larger than 10 .

The method makes a certain number of assumptions, as follows:

(a) The populations of different levels belonging to the same spectroscopic term are approximately proportional to their statistical weights.

(b) The self absorption of lines of different multiplets is always the same function of the optical thickness. In the optical region studied by us, our analysis is mainly based on lines whose lower levels are both even and metastable, while the upper levels are odd. The processes of excitation are supposed to be similar. If such a situation exists, we may expect that effects of inhomogeneities in the emitting region, affect the different lines in almost the same way.

(c) Selective excitation mechanisms, and particularly pumping of populations of upper levels of emision lines because of wavelength coincidences with other strong emission lines, are fairly infrequent and can be neglected for most lines. Such an assumption would be quite dangerous in the ultraviolet wavength range (Eriksson et al. 2004).

Other assumptions can often be made, as in this paper, when applying the method:

(d) The populations of different collisionally excited even lower terms of $\mathrm{Fe}^{+}$follow roughly Boltzmann's law. In fact one can expect LTE at least for those having a lower excitation potential. The only relevant calculations of Verner et al. (2002) for emission by the B+D Weigelt blobs of $\eta$ Car, with an estimated electron density of the order of only $10^{6} \mathrm{~cm}^{-3}$, suggest that Boltzmann's law is obeyed up to $2.3 \mathrm{eV}$, with deviations as a function of excitation potential for higher metastable levels. Our lines are formed in a circumstellar region, with a presumably much higher electron density and larger collisional excitations and deexcitation rates of the metastable levels, as indeed is consistent with the determined column densities and limits on the size of the line emitting region, given below (see Sect. 5.2, for what may happen, if this assumption is relaxed).

(e) The populations of the upper odd levels are not in LTE, but we suppose that they tend to cluster around a Boltzmann law like distribution. Our analysis of HD 45677 is consistent with such an assumption for levels, which are not highly excited (see below), irrespective of what the dominant excitation mechanism may be. Let us note that the Verner et al. (2002) $\eta$ Car analysis, indicates departure coefficients, which tend to be mostly a function of the excitation potential.

\subsection{Fe ॥ permitted emission lines}

We first consider the narrow emission components of Fe II. For the analysis we adopt Kurucz' (1993) oscillator strengths. In order to look for self-absorption effects, we have analysed the narrow component line fluxes of individual $\mathrm{Fe}$ II multiplets in the $\left[\log g f \lambda, \log \frac{F \lambda^{3}}{g f}\right]$ diagram, where the quantity $\frac{F \lambda^{3}}{g f}$ is the normalized flux of an individual emission line, and $g f \lambda$ is proportional, for a given multiplet, to the optical thickness at the
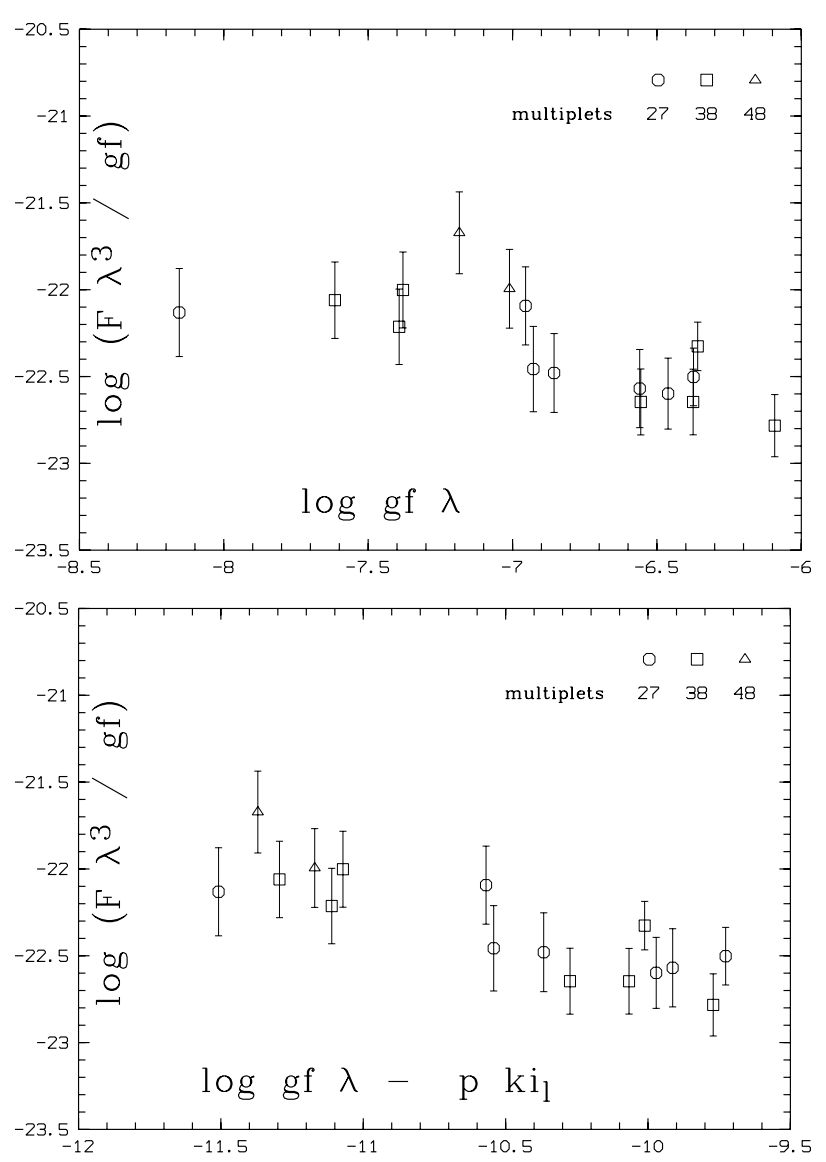

Fig. 6. Upper panel: $\log$ normalized fluxes versus $\log g f \lambda$ plots for the narrow components of Fe II multiplets 27 (circle), 38 (square), 48 (triangle) with common upper term $\mathrm{z}^{4} \mathrm{D}^{0}$ in the 1999 spectrum of HD 45677. Lower panel: $\log$ normalized fluxes versus $\log g f \lambda-p \chi_{1}$ plots of multiplets 27,38 and 48 . The multiplets are horizontally shifted according to the population ratio of their lower terms by $p=1.3$.

line centre (see Viotti et al. 2000). This procedure requires of course the first of the above assumptions (a).

In the upper panel of Fig. 6 we show the diagram for multiplets 27,38 and 48 that have $\mathrm{z}^{4} \mathrm{D}^{0}$ as common upper term. In the diagram, if the lines were optically thin, the normalized emission line flux should be constant within a multiplet. We note on the contrary that the strongest lines of multiplets 27 and 38 have a normalized flux lower than that of the weaker multiplet lines. This indicates that they are affected by a certain amount of self-absorption. It should also be noticed that we have not found for any multiplet any systematic trend, within the measurement errors of the line fluxes, with respect to the quantum number $J$, which would suggest a contradiction with assumption (a), about level populations within the upper and lower terms of each multiplet. Anyhow, a thourough study of this effect will be difficult for such complex line profiles, even with much higher quality material.

The bend of these diagrams helps to determine the relative population of the lower terms of the multiplets, by their horizontal shifts with respect to each other by an amount equal to the logarithm of this population ratio (e.g. Kotnik-Karuza et al. 2002). This has been performed in the $\left[\log g f \lambda-p \chi_{1}, \log \frac{F \lambda^{3}}{g f}\right]$ 
diagram (lower part of Fig. 6) where the multiplets have been overlapped assuming a power law with exponent $-p$. A value of $\left.p=1.3 \pm 0.4 \mathrm{eV}^{-1}\right)$ is derived for the exponent of the power law of the lower terms population of the multiplets 27,38 and 48 .

Similarly, by comparing the 1999 diagrams for multiplets 28, 37 and 49 , having $\mathrm{z}^{4} \mathrm{~F}^{0}$ as common upper term, we derive a mean lower level population gradient of $p=1.0 \pm 0.4 \mathrm{eV}^{-1}$.

In general these results are fairly uncertain because of the small energy range of the lower terms, and the same is the case of multiplets with common lower term, which cannot be properly used to derive the mean excitation of the upper terms because of the small energy range of our data. In order to overcome this problem, we have assumed that all the multiplets fit a common theoretical self-absorption curve $Q(\tau)$, and have used the least squares method to determine the parameters $p, X_{\mathrm{c}}, q$, $Y_{\mathrm{c}}$, needed to overlap the lines of all multiplets to the theoretical curve in an $\left[X-X_{\mathrm{c}}, Y-Y_{\mathrm{c}}\right]$ diagram, where they correspond to the following physical definitions:

$$
\begin{aligned}
& X=\log g f \lambda-p\left(\chi_{1}-\chi_{\mathrm{c}}\right) \\
& Y=\log \frac{F \lambda^{3}}{g f}+q\left(\chi_{\mathrm{u}}-\chi_{\mathrm{c}}\right)
\end{aligned}
$$

$X_{\mathrm{c}}=1.576-\log \frac{N_{\mathrm{c}}}{g_{\mathrm{c}}}+\log v_{\mathrm{c}}$

$Y_{\mathrm{c}}=\log \frac{S^{\prime}}{d^{2}}+\log \frac{N_{\mathrm{c}}}{g_{\mathrm{c}}}+w-\log \frac{2 \pi e^{2} h}{m_{\mathrm{e}}}$

and $\log \frac{2 \pi e^{2} h}{m_{\mathrm{e}}}=16.977$ in cgs units.

The quantities $\chi_{1}$ and $\chi_{\mathrm{u}}$ are the excitation potentials (in $\mathrm{eV}$ ) of the lower and upper levels of each transition, $\chi_{\mathrm{c}}$ is a characteristic excitation potential taken different from $0 \mathrm{eV}$ in the case of Fe II permitted lines (and equal to $2.75 \mathrm{eV}$ ) in order to avoid problems of the populations of the higher metastable levels, whose possible large departures from LTE are pointed out by Verner et al. (2002). The unknown parameters $p$ and $q$ describe the mean population distributions of the lower and upper terms, respectively (Muratorio et al. 1992). In this procedure we assume the first of the above assumtions (a), as verified in each multiplet $\left[\log g f \lambda, \log \frac{F \lambda^{3}}{g f}\right]$ diagram, and that all the lower and upper levels follow a Boltzmann-type law with excitation temperatures $T_{1}$ and $T_{\mathrm{u}}$ of 5040/p and 5040/q, respectively.

The zero points $X_{\mathrm{c}}$ and $Y_{\mathrm{c}}$, are related to the $\mathrm{Fe}^{+}$column density $\frac{N_{\mathrm{c}}}{g_{\mathrm{c}}}$ at the arbitrary choosen level $\chi_{\mathrm{c}}$, taken to be equal to $2.75 \mathrm{eV}$ for Fe II permitted lines, and to the projected extension of the emitting volume $S^{\prime}\left(S^{\prime}=S \cos i\right)$, through the Eqs. (3) and (4) (Friedjung \& Muratorio 1987; Baratta et al. 1998). The column density $\frac{N_{c}}{g_{\mathrm{c}}}$ also depends on the parameter $v_{\mathrm{c}}$ (Eq. (3), which is the velocity broadening of the opacity profile (see Sect. 5.2). For the theoretical SAC function $Q(\tau)$ we adopted the analytical expression for a Gaussian line profile and an homogeneous emitting envelope given by Friedjung \& Muratorio (1987).

All the quantities are in cgs units. The term w in Eq. (4) describes the overall deviation of the population of the upper (odd) levels of the $\mathrm{Fe}$ II transitions with respect to the population of the lower (even) ones. If we assume that the odd and

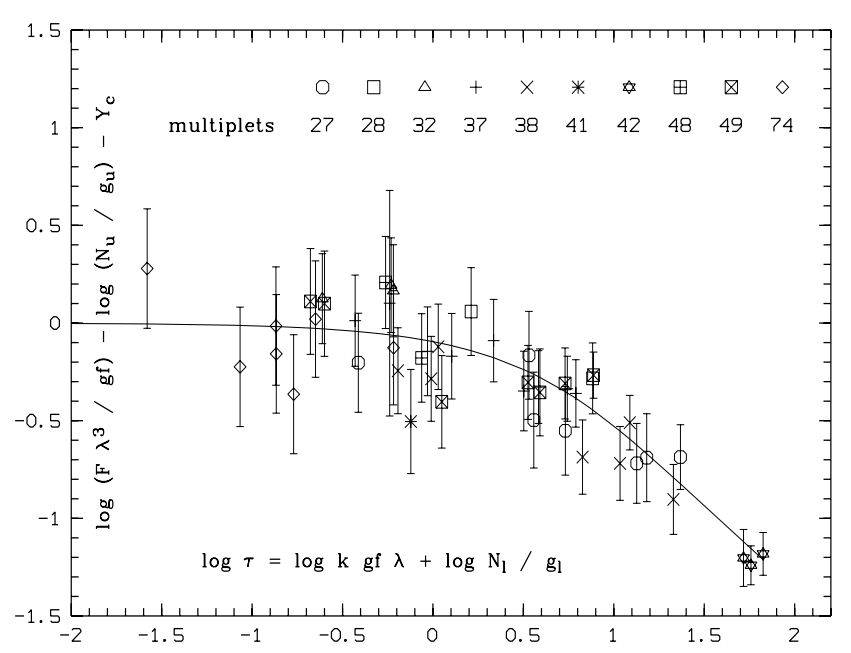

Fig. 7. Global SAC of the narrow emission components in the 1999 spectrum of HD 45677. Results from the least squares overlapping of multiplets 27, 28, 32, 37, 38, 41, 42, 48, 49 and 74. The representative points are shifted horizontally and vertically according to a least squares fitting procedure by log level population corresponding to excitation temperatures of $3900 \mathrm{~K}$ and $3150 \mathrm{~K}$ for the lower and upper terms respectively.

even level population laws link up at an intermediate $\bar{\chi}$, the term turns out to be equal to $w=\left(\bar{\chi}-\chi_{\mathrm{c}}\right)(q-p)$, where for the case of Fe II one can take $\bar{\chi}=4.72 \mathrm{eV}$. Since we expect the odd terms to be largely underpopulated with respect to the even ones (see e.g. Fig. 13 in Verner et al. 2002), this value of w should be taken as an upper limit, which corresponds to a lower limit to $S^{\prime}$.

Figure 7 shows the result of the least squares fit of the dereddened fluxes (with error bars) of 49 narrow components of the $\mathrm{Fe}$ II lines measured in the 1999 spectrum of HD 45677. The high excitation $\left(\chi_{\mathrm{u}}>7.5 \mathrm{eV}\right) \mathrm{Fe}$ II lines have not been included in the fit, and will be discussed separately in Sect. 3.2.

As expected, in Fig. 7 multiplet 74 emission lines lie on the optically thin branch of the SAC, while the very strong lines of multiplet 42 lines are the most optically thick ones (central line opacity of about 70 ). Note that many multiplets lie around the bend of the $\operatorname{SAC}(\log \tau \simeq 0)$.

The fit gives a mean lower level population $\mathrm{p}$ corresponding to an excitation temperature $T_{1}$ of $3900_{-600}^{+900} \mathrm{~K}$, in fairly good agreement with the values previously found by considering two groups of multiplets having common upper terms. The resulting mean upper level population parameter $q$ corresponds to a mean excitation temperature $T_{\mathrm{u}}$ of $3150_{-300}^{+350} \mathrm{~K}$, much smaller than that of the lower levels. Let us note that the modelling of Verner et al. (2002) for two of the Weigelt blobs of $\eta$ Car predicted electron temperatures going down to $5000 \mathrm{~K}$.

We have applied this analysis also to the narrow $\mathrm{Fe}$ II emission components in the 1995 and 2001-2002 spectra of HD 45677. The derived best fit parameters for the three epochs are summarized in Table 6. One may note in the table that the derived $Y_{\mathrm{c}}$ parameters are very different from one epoch to another. This cannot be totally ascribed to a variation of the size of the $\mathrm{Fe}$ II emitting region, to which the $Y_{\mathrm{c}}$ parameter is related, since, as noted in Sect. 2.2, the spectrum did not largely 
Table 6. SAC fit parameters for the permitted Fe II narrow component lines. $p\left(\mathrm{eV}^{-1}\right), X_{\mathrm{c}}, q\left(\mathrm{eV}^{-1}\right)$ and $Y_{\mathrm{c}}$ are fitting parameters, $\chi^{2}$ is a measure of the fitting precision.

\begin{tabular}{cccc}
\hline \hline year & 1995 & 1999 & 2001 \\
\hline$p$ & $1.32 \pm 0.33$ & $1.28 \pm 0.23$ & $0.94 \pm 0.31$ \\
$X_{\mathrm{c}}$ & $-7.27 \pm 0.09$ & $-7.52 \pm 0.08$ & $-7.37 \pm 0.11$ \\
$q$ & $1.79 \pm 0.19$ & $1.60 \pm 0.16$ & $1.28 \pm 0.17$ \\
$Y_{\mathrm{c}}$ & $-17.00 \pm 0.54$ & $-17.40 \pm 0.47$ & $-18.37 \pm 0.47$ \\
$N$ lines & 50 & 50 & 28 \\
\hline red. $\chi^{2}$ & 0.65 & 0.45 & 0.34 \\
\hline
\end{tabular}

Table 7. SAC fit parameters for the permitted Fe II broad component lines. See Table 6 and text for definitions. * parameter taken equal to the narrow components one as no convergence is obtained.

\begin{tabular}{cccc}
\hline \hline year & 1995 & 1999 & 2001 \\
\hline$p$ & $1.32^{*}$ & $1.28^{*}$ & $0.94^{*}$ \\
\hline$X_{\mathrm{c}}$ & $-6.97 \pm 0.14$ & $-7.03 \pm 0.11$ & $-7.29 \pm 0.18$ \\
$q$ & $0.99 \pm 0.29$ & $1.50 \pm 0.26$ & $1.53 \pm 0.26$ \\
$Y_{\mathrm{c}}$ & $-19.35 \pm 0.82$ & $-17.84 \pm 0.75$ & $-17.84 \pm 0.74$ \\
$N$ lines & 49 & 49 & 28 \\
red. $\chi^{2}$ & 0.45 & 0.41 & 0.33 \\
\hline
\end{tabular}

vary during 1995-2002. Actually, the fitting value of $Y_{\mathrm{c}}$ is correlated with the $q$ parameter, so that a small error on $q$ results in a large change in $Y_{\mathrm{c}}$. In view of this point, as also discussed above, in this paper we shall mostly concern ourselves with the 1999 spectrum of HD 45677, and leave the problem of the spectral variation to a forthcoming paper.

The same procedure has been applied to the Fe II broad emission components components. Figure 8 shows the global SAC of the Fe II broad emissions in the 1999 spectrum of HD 45677 obtained from the least squares overlapping of ten Fe II multiplets. The fluxes of the broad components are less precisely determined than the narrow component ones, and the scatter of their SAC curves is higher, respectively.

The fitting parameters of the Fe II broad emission components are given in Table 7.

The SAC analysis of the broad components in 1995 and 2001-2002 is more uncertain, due to the lower spectral resolution in 1995, which results in a less precise determination of the broad component fluxes, and to the smaller set of data in 20012002. Despite the lack of convergence for the $p$ value in 1999 , the parameters, summarized in Table 7, appear fairly similar to the ones derived from the narrow emission components except for the 1995 data, despite the too small $q$ value derived.

\subsection{High excitation Fe ॥ permitted lines}

In the spectrum of HD 45677 we have identified many emission lines arising from high energy levels $\left(\chi_{\mathrm{u}} \geq 7.5 \mathrm{eV}\right)$, that have not been included in the previous SAC analysis. Figure 9 shows the position of the narrow components of these lines with respect to the SAC derived from the narrow components of the lower excitation $\mathrm{Fe}$ II lines. Indeed, plotting these lines in the same graph may present a difficulty, since we cannot adopt for these lines the same excitation temperature of the lower

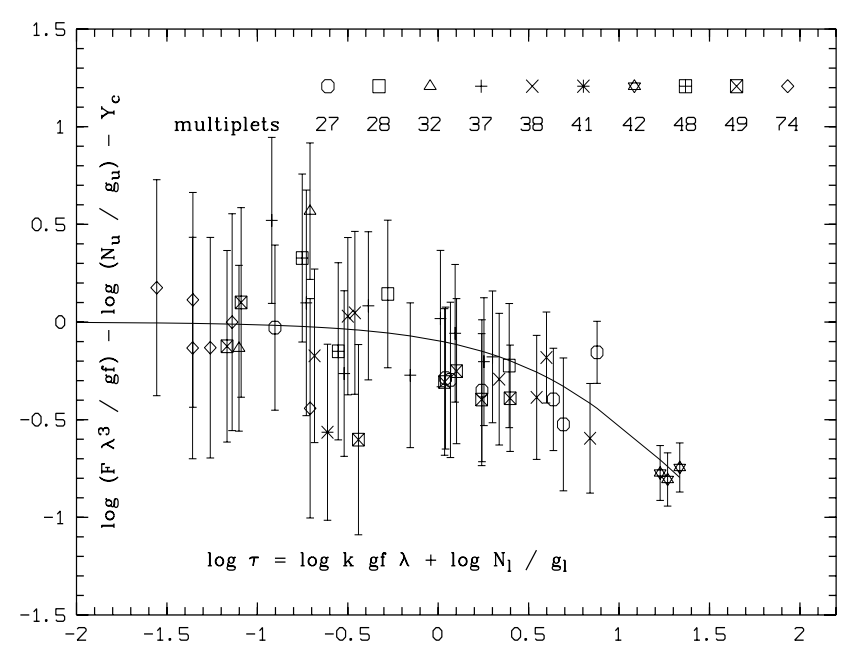

Fig. 8. SAC of broad components of multiplets 27, 28, 32, 37, 38, 41, 42, 48, 49 and 74 of Fe II in 1999. The representative points are shifted horizontally and vertically by log level population corresponding to excitation temperatures of respectively $3900 \mathrm{~K}$ and $3400 \mathrm{~K}$.

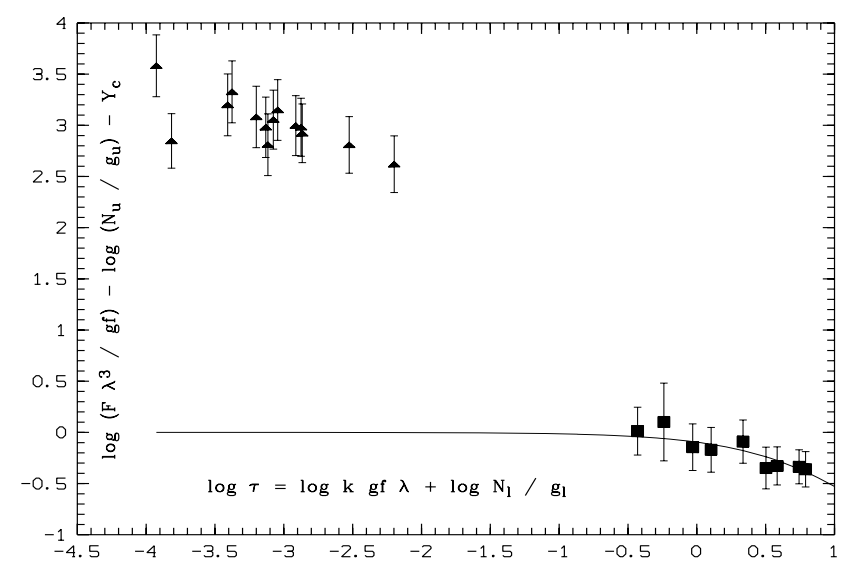

Fig. 9. Position of the high excitation Fe II lines (filled triangles) in the 1999 spectrum of HD 45677 with respect to the global SAC of Fig. 7. The multiplet 37 lines (filled squares) are shown for comparison.

excitation Fe II lines, or even define an excitation temperature for the upper levels, as they certainly are far from equilibrium. However, it is clear from the figure that, whatever level excitation law is adopted, the high excitation lines are 2-3 orders of magnitude stronger than if they were excited by the same mechanism as the lower excitation permitted lines. The values displayed in Table 8 are a clear indication of an overpopulation of the upper levels, as also appears in Fig. 17. This will be discussed in Sect. 5.1.

\subsection{Fe ॥ forbidden emission lines}

Now we analyse the narrow and broad components of the forbidden lines of ionized iron observed in the 1999 spectrum of HD 45677. We adopted the [Fe II] transition probabilities of Quinet et al. (1996), and took for simplicity $\chi_{\mathrm{c}}=0 \mathrm{eV}$.

The plot of [Fe II] individual multiplets in the $\left[\log g f \lambda, \log \frac{F \lambda^{3}}{g f}\right]$ diagram shows a systematical downward shift of multiplets arising from higher excitation terms, 
Table 8. For each high excitation line we give: (1) the laboratory wavelength, (2) upper and lower term of the transition, (3) upper and lower term excitation potential, (4) logarithm of oscillator strength, (5) log ratio of observed flux to calculated flux according to the lower excitation lines parameters.

\begin{tabular}{|c|c|c|c|c|}
\hline Wavelength & $\begin{array}{l}\text { upper Term } \\
\text { lower Term }\end{array}$ & $\begin{array}{l}\chi_{\text {up }} \\
\chi_{\text {low }}\end{array}$ & $\log g f$ & $\log \frac{F_{\text {obs }}}{F_{\text {calc }}}$ \\
\hline 6158.019 & $\begin{array}{l}(3 \mathrm{G}) 4 \mathrm{p} \times 4 \mathrm{~F} \\
(3 \mathrm{~F}) 4 \mathrm{~s} \mathrm{c} 4 \mathrm{~F}\end{array}$ & $\begin{array}{l}8.23 \\
6.22\end{array}$ & -3.09 & 4.1 \\
\hline 6160.755 & $\begin{array}{l}(3 \mathrm{H}) 4 \mathrm{p} \mathrm{z} 4 \mathrm{H} \\
(1 \mathrm{~F}) 4 \mathrm{~s} \mathrm{c} 2 \mathrm{~F}\end{array}$ & $\begin{array}{l}7.58 \\
5.57\end{array}$ & -3.63 & 3.5 \\
\hline 6233.534 & $\begin{array}{l}d 54 s 2 \mathrm{c} 4 \mathrm{D} \\
(5 \mathrm{D}) 4 \mathrm{p} z 4 \mathrm{~F}\end{array}$ & $\begin{array}{l}7.47 \\
5.48\end{array}$ & -2.94 & 2.7 \\
\hline 6248.898 & $\begin{array}{l}d 54 s 2 c 4 D \\
\text { (5D) } 4 p \text { z } 4 D\end{array}$ & $\begin{array}{l}7.49 \\
5.51\end{array}$ & -2.70 & 2.9 \\
\hline 6317.983 & $\begin{array}{l}d 54 s 2 c 4 D \\
(5 D) 4 p \text { z } 4 D\end{array}$ & $\begin{array}{l}7.47 \\
5.51\end{array}$ & -1.99 & 2.6 \\
\hline 6371.125 & $\begin{array}{l}d 54 s 2 c 4 D \\
(5 D) 4 p \text { z } 4 F\end{array}$ & $\begin{array}{l}7.49 \\
5.55\end{array}$ & -3.56 & 2.8 \\
\hline 6383.722 & $\begin{array}{l}d 54 s 2 c 4 D \\
(5 D) 4 p \text { z } 4 D\end{array}$ & $\begin{array}{l}7.49 \\
5.55\end{array}$ & -2.27 & 2.7 \\
\hline 6385.451 & $\begin{array}{l}d 54 s 2 c 4 D \\
(5 D) 4 p \text { z } 4 D\end{array}$ & $\begin{array}{l}7.49 \\
5.55\end{array}$ & -2.62 & 2.9 \\
\hline 6442.955 & $\begin{array}{l}d 54 s 2 c 4 D \\
(5 D) 4 p \text { z } 4 F\end{array}$ & $\begin{array}{l}7.47 \\
5.55\end{array}$ & -2.88 & 2.9 \\
\hline 6455.837 & $\begin{array}{l}d 54 s 2 c 4 D \\
\text { (5D) } 4 p \text { z } 4 D\end{array}$ & $\begin{array}{l}7.47 \\
5.55\end{array}$ & -2.95 & 3.0 \\
\hline 6482.204 & $\begin{array}{l}(3 \mathrm{G}) 4 \mathrm{p} \times 4 \mathrm{G} \\
(3 \mathrm{~F}) 4 \mathrm{~s} \text { c4F }\end{array}$ & $\begin{array}{l}8.13 \\
6.22\end{array}$ & -2.27 & 3.3 \\
\hline 6491.246 & $\begin{array}{l}d 54 s 2 c 4 D \\
\text { (5D) } 4 p \text { z } 4 D\end{array}$ & $\begin{array}{l}7.49 \\
5.58\end{array}$ & -2.79 & 3.0 \\
\hline 6493.035 & $\begin{array}{l}d 54 s 2 c 4 D \\
\text { (5D)4p z4D }\end{array}$ & $\begin{array}{l}7.49 \\
5.58\end{array}$ & -2.58 & 2.9 \\
\hline 6506.333 & $\begin{array}{l}d 54 s 2 c 4 D \\
(5 D) 4 p \text { z } 4 F\end{array}$ & $\begin{array}{l}7.49 \\
5.59\end{array}$ & -3.11 & 3.1 \\
\hline 6517.018 & $\begin{array}{l}d 54 s 2 c 4 D \\
(5 D) 4 p \text { z } 4 D\end{array}$ & $\begin{array}{l}7.49 \\
5.58\end{array}$ & -2.76 & 3.1 \\
\hline
\end{tabular}

that can be used to determine their upper level population gradient $q$. In the strongest multiplets there is also an indication of the most intense lines having a smaller normalised flux.

We have overlapped the [Fe II] multiplets in the $\left[X-X_{\mathrm{c}}, Y-Y_{\mathrm{c}}\right]$ plane, in the same way as we did for permitted $\mathrm{Fe}$ II lines, with the least squares method, assuming that the ground and lower $\mathrm{Fe}^{+}$levels have the same excitation temperature (i.e., $p=q=5040 / T_{f}$ ). The derived SAC diagram shown in Fig. 10 corresponds to a best fit excitation temperature of the metastable levels $(0-4 \mathrm{eV})$ of $T_{f}=10500 \pm 1000 \mathrm{~K}$. Actually, the upper [Fe II] levels are the same as the lower levels of the permitted transitions treated above. Nevertheless, the fact that two such different excitation temperatures have been derived, added to the smaller $F W H M$ value of the [Fe II], $25 \mathrm{~km} \mathrm{~s}^{-1}$ against $45 \mathrm{~km} \mathrm{~s}^{-1}$ for Fe II permitted lines, is strongly suggestive of two different formation regions. The results for the three epochs are summarised in Table 9.

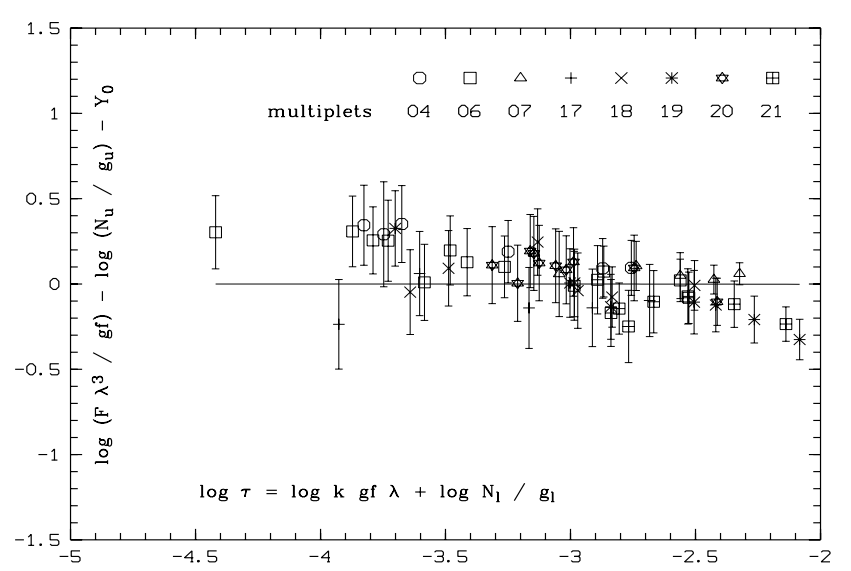

Fig. 10. SAC diagram of narrow components of multiplets 4F, 6F, 7F, $17 \mathrm{~F}, 18 \mathrm{~F}, 19 \mathrm{~F}, 20 \mathrm{~F}$ and $21 \mathrm{~F}$ of [Fe II] in 1999. The same mean level excitation temperature of $10500 \mathrm{~K}$ has been used for both lower and upper levels. The observational points are interpolated with horizontal line.

Table 9. SAC fit parameters for the [Fe II] narrow components. $q\left(\mathrm{eV}^{-1}\right)$ and $Y_{f}$ are fitting parameters. $\bar{Y}$ is $Y_{f}$ corrected for $q$ at a mean $3 \mathrm{eV}$ excitation potential. $\log \bar{N}(\mathrm{H})$ is the logarithm of the total number of Hydrogen atom in the emitting volume.

\begin{tabular}{cccc}
\hline \hline year & 1995 & 1999 & 2001 \\
\hline$q$ & $0.44 \pm 0.05$ & $0.47 \pm 0.05$ & $0.57 \pm 0.08$ \\
$Y_{\mathrm{f}}$ & $-15.51 \pm 0.16$ & $-15.41 \pm 0.13$ & $-15.20 \pm 0.25$ \\
$\bar{Y}$ & -16.83 & -16.82 & -16.91 \\
$T_{f}$ & $11500 \pm 1000$ & $10500 \pm 1000$ & $9000 \pm 2000$ \\
$\log \bar{N}(\mathrm{H})$ & $49.9 \pm .2$ & $50.0 \pm .1$ & $50.2 \pm .3$ \\
$N \operatorname{lines}$ & 85 & 80 & 31 \\
red. $\chi^{2}$ & 0.68 & 0.82 & 0.94 \\
\hline
\end{tabular}

Let us point in Table 9 that the variation in the different epochs of the $T_{f}$ and $Y_{f}$ parameters are much smaller than those derived for the permitted lines, probably as the result of the smaller uncertainties. The value, at the mean upper level excitation potential of our sample lines $\overline{\chi_{\mathrm{u}}}=3.0 \mathrm{eV}$, of the quantity $\bar{Y}=-p \times \overline{\chi_{\mathrm{u}}}+Y_{f}$ is smaller by a factor .8 in 2001 corresponding approximately to the small flux decrease between 1999 and 2001-2002 noted in Sect. 2.2.

As for the broad components of the [Fe II] emission lines, the strength of the better measured lines seems to suggest by itself an emitting region with properties comparable to those of the narrow components discussed above, this is confirmed by the fitting values given in Table 10 .

\section{4. $\mathrm{Cr}$ II emission lines}

The analysis of the narrow and broad $\mathrm{Cr}$ II multiplet 44 components, indicates a moderate optical thickness. Here again we adopted $\chi_{\mathrm{c}}=0 \mathrm{eV}$ in the equations defined in Sect. 3.1. Due to the low number of $\mathrm{Cr}$ II lines observed in the other multiplets we have derived the SAC parameters taking the same excitation temperatures for the lower and upper terms of both the narrow and broad components. 


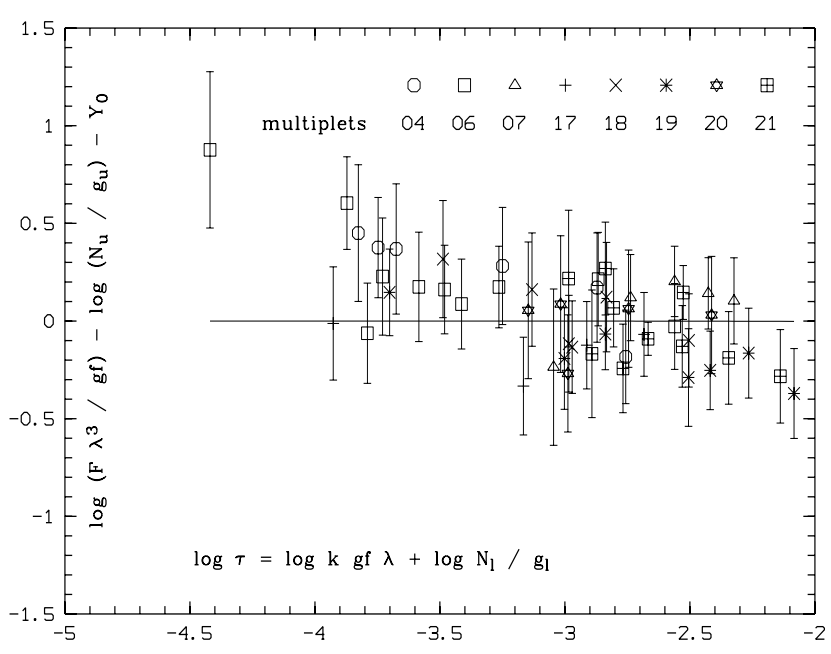

Fig. 11. SAC diagram of broad components of multiplets $4 \mathrm{~F}, 6 \mathrm{~F}, 7 \mathrm{~F}$, $17 \mathrm{~F}, 18 \mathrm{~F}, 19 \mathrm{~F}, 20 \mathrm{~F}$ and $21 \mathrm{~F}$ of $[\mathrm{Fe} \mathrm{II}]$ in 1999 . The representative points are shifted horizontally and vertically by log level population corresponding to an excitation temperature of $8500 \mathrm{~K}$.

Table 10. SAC fit parameters for the [Fe II] broad components.

\begin{tabular}{cccc}
\hline \hline year & 1995 & 1999 & 2001 \\
\hline$q$ & $0.50 \pm 0.20$ & $0.64 \pm 0.09$ & $0.48 \pm 0.18$ \\
$Y_{f}$ & $-15.95 \pm 0.60$ & $-15.38 \pm 0.26$ & $-15.94 \pm 0.53$ \\
$\bar{Y}$ & -17.45 & -17.30 & -17.38 \\
$T_{f}$ & $10000 \pm 3000$ & $8000 \pm 1500$ & $10500 \pm 3000$ \\
$\log \bar{N}(\mathrm{H})$ & $49.5 \pm .6$ & $50.0 \pm .3$ & $49.5 \pm .5$ \\
$N \operatorname{lines}$ & 61 & 63 & 28 \\
red. $\chi^{2}$ & 2.0 & 1.23 & 1.45 \\
\hline
\end{tabular}

The SACs plotted in Figs. 12 and 13 correspond to the parameters given in Table 11.

\section{Line profiles}

The two-component profile of the emission lines is suggestive of line formation in two regions with different velocity broadening in the observer's line-of-sight. In B[e] stars, of which HD 45677 is one important representative, low excitation emission lines, like among others $\mathrm{Fe}$ II and $\mathrm{Cr}$ II, are generally thought to be formed in an equatorial disk around the B star, while the higher temperature emission lines are thought to be emitted in a polar wind. Zickgraf (2003) recently studied the kinematical structure of $\mathrm{B}[\mathrm{e}]$ star envelopes based on high resolution profiles of permitted and forbidden emission lines, and compared them with theoretical profiles emerging from an optically thin latitude-dependent expanding stellar wind and an equatorial opaque dust ring. He observed in his 1986-88 CATCES spectra of HD 45677 a marginal line split of $6 \mathrm{~km} \mathrm{~s}^{-1}$ for the [O I] $6300 \AA$ A emission line, while [Fe II] and [N II] were single peaked. He interpreted his observations with line formation in a wide opening angle polar wind seen nearly pole-on.

In HD 45677 the profile of the emission lines do not show the double peaked structure typical of line emission from an equatorial rotating disk seen at high inclinations. The narrow

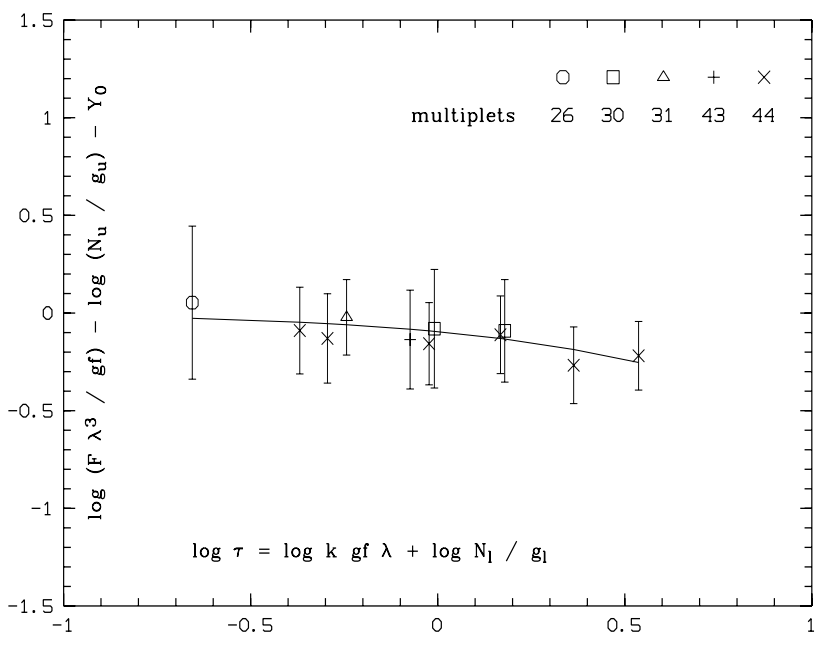

Fig. 12. Self Absorption Curve for the narrow components of $\mathrm{Cr}$ II emission lines.

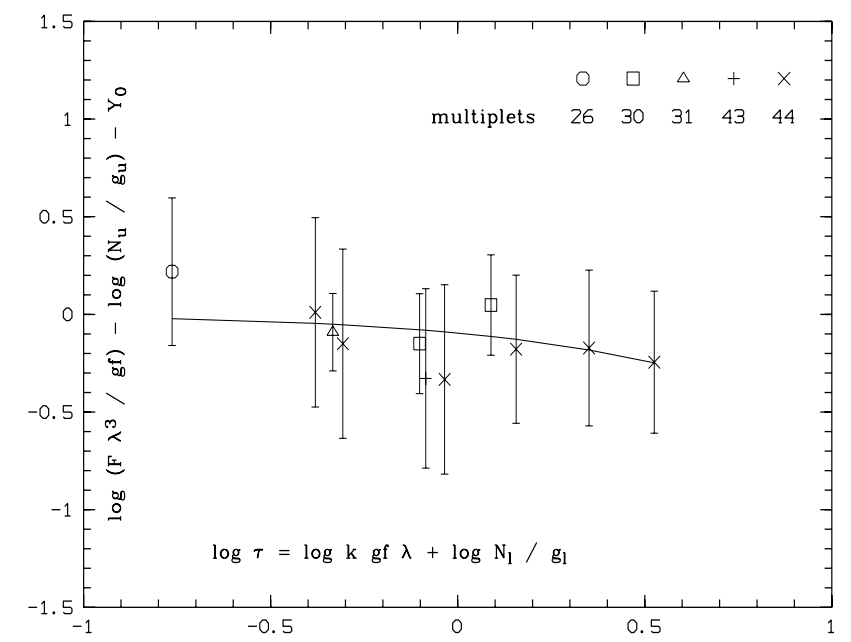

Fig. 13. Self Absorption Curve for the broad components of the $\mathrm{Cr}$ II emission lines.

components of strongest permitted emission lines are split by a slightly longward displaced $\left(\sim+3 \mathrm{~km} \mathrm{~s}^{-1}\right)$ dip which we attribute instead to absorption from matter flowing towards the star. A central absorption with the same radial velocity is also present in the Na I yellow doublet, and in the Mg II $4481 \AA$ line. No line splitting is seen in the forbidden lines. Another important feature of HD 45677 is the narrower profile of the forbidden [Fe II] lines, suggesting line formation in regions with a smaller radial velocity range, like for instance the outer parts of a rotating disk in which the inner, denser and faster rotating parts produce the permitted emission lines.

In HD 45677 the velocity broadening of the $\mathrm{Si}$ II photospheric lines $\left(\leq \sim 70 \mathrm{~km} \mathrm{~s}^{-1}\right.$, Israelian et al. 1996) should indicate either a relatively low stellar rotational velocity, or a low inclination angle $i$ of a fast rotating star. If this is the case, the narrow emission components could arise from a dense, fast rotating disk seen nearly face-on. If one assumes that matter in the disk is rotating at the Keplerian velocity, the narrower [Fe II] emission lines should be formed in the outer (and less dense) rings of the disk. In such a framework, the broad components of the $\mathrm{Fe}$ II and [Fe II] emission lines, that, as discussed above, 
Table 11. SAC fit parameters for the narrow and broad component lines of CrII in 1999.

\begin{tabular}{ccc}
\hline \hline component & narrow & broad \\
\hline$p=q$ & $0.82 \pm 0.15$ & $0.43 \pm 0.28$ \\
$X_{\mathrm{c}}$ & $-8.63 \pm 0.60$ & $-7.03 \pm 1.00$ \\
$Y_{\mathrm{c}}$ & $-18.42 \pm 0.98$ & $-20.87 \pm 1.85$ \\
$N$ lines & 11 & 11 \\
red. $\chi^{2}$ & 0.07 & 0.19 \\
\hline
\end{tabular}

approximately emit a total amount of radiative power comparable to that of the narrow components, should be formed in region(s) with a large radial velocity range in the observer's line-of-sight. This could be identified as being a rather massive stream of matter flowing from (or to) the stellar polar regions.

We geometrically model the emitting region of HD 45677 in order to synthetise simultaneously both the observed profiles of the [Fe II] emission lines and the Fe II ones.

As discussed above, the model of a disk seen nearly edgeon proposed for this star for instance by Swings (1973) or de Winter et al. (1997) besides the fact that it would imply a too small star rotational velocity, would generate emission line profiles with the typical double-peaked profile totally different from the ones observed.

The bimodal model currently adopted for B[e] stars supposes different physical conditions in the dense disk medium, where the emission lines of the lower excitation species such as Fe II are supposed to be produced, and in the polar wind or streams, where lines from higher excitation species are emitted (Swings 1973; Zickgraf 1998). We nevertheless took this configuration, namely an edge-on disk and a polar wind confined in a cone having the same physical conditions (temperature and density), into consideration. The most similar profile was obtained with a $24^{\circ}$ opening angle disk inclined $85^{\circ}$ on the sky plane, in which the wide component is emitted, and a $30^{\circ}$ cone wind for wich we had to suppose a $100 \mathrm{~km} \mathrm{~s}^{-1}$ decreasing velocity wind to get the narrow component. The computed profile is shown in Fig. 14. But the result is unsatisfying (too sharp).

In order to get better results we had to suppose that the narrow components are produced in a nearly pole-on optically thin disk. We got satisfying results taking an inclination to the sky plane of $12^{\circ}$, and as a first step a keplerian rotational velocity taken to be $340 \mathrm{~km} \mathrm{~s}^{-1}$ at the star surface. With such an inclination and velocity we are in agreement with the $v \sin i=70 \mathrm{~km} \mathrm{~s}^{-1}$ determination of Israelian et al. (1996). Such a region of line formation, would, when seen nearly pole on, resemble Kastner's static model.

To get the broad line components we had to suppose the existence of a flow falling on the disk (or alternatively escaping from the disk), whose material is incorporated into (or alternatively ejected from) the disk material at the Keplerian velocity in each point.

With such a configuration we can reproduce at the same time as well the permitted (Fig. 15) as the forbidden (Fig. 16) Fe II line profiles, by just playing on the distance to the star of the emitting zone.

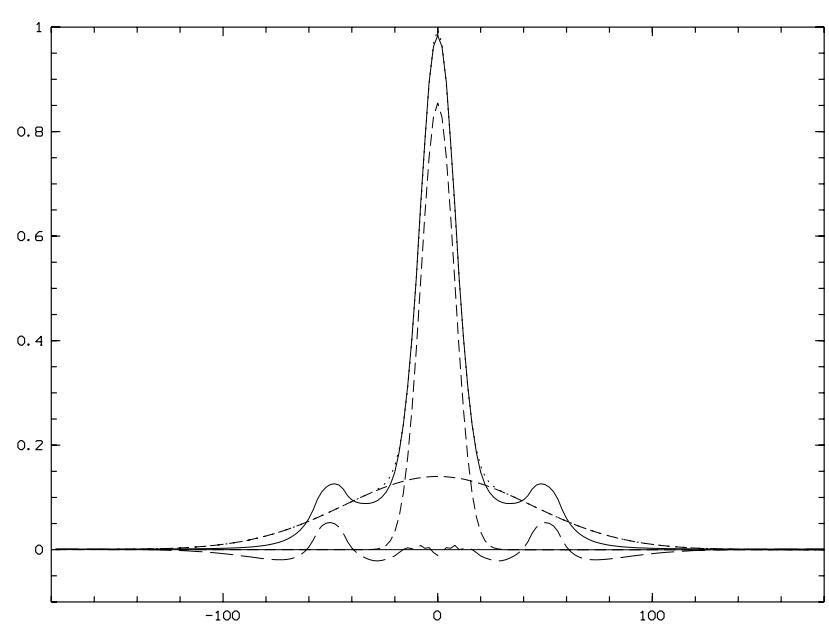

Fig. 14. Histogram of the emitting material radial velocities of an optically thin disk plus polar wind. The disk is inclined $85^{\circ}$ to the sky plane with a total opening angle of $24^{\circ}$. The velocity in the disk is $150 \mathrm{~km} \mathrm{~s}^{-1}$ at the photospheric radius $r=1$, and the law is Keplerian. The polar wind is emitted in a $30^{\circ}$ cone with a velocity of $100 \mathrm{~km} \mathrm{~s}^{-1}$ at the pole and decreases exponentialy. In this figure and in Figs. 15 and 16, the synthetised profile and the continuum are plotted as a solid line, each Gaussian component as a short dashed line, the total fit profile as a dotted line and the residual flux as a long dashed line.

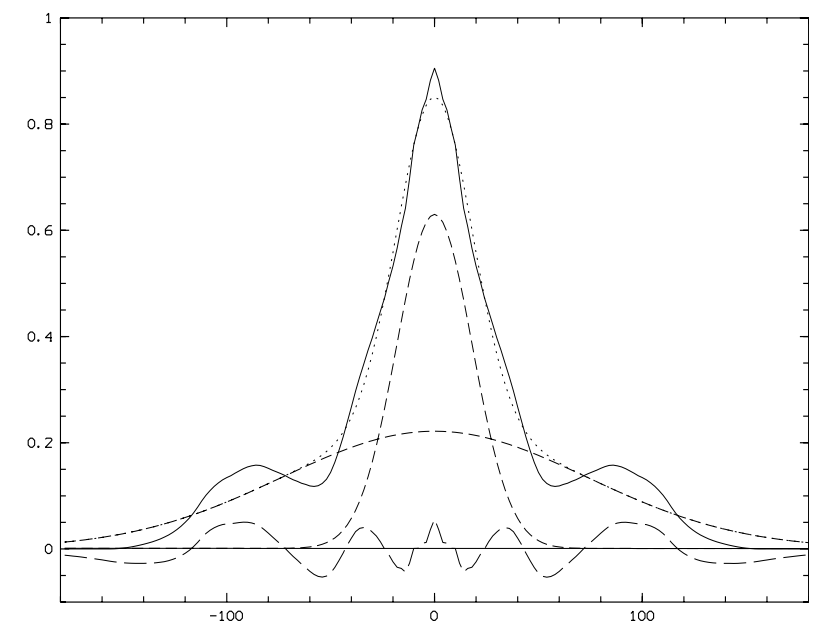

Fig. 15. Theoretical profile corresponding to the histogram of the emitting material radial velocities of an optically thin Keplerian disk inclined $12^{\circ}$ on the sky plane. An accreting wind generates the broad component. The total fit profile coincides with the broad component for large velocities, and with the sum of the components for small velocities. The component FWHM are the ones derived in Sect. 2.2. The Keplerian velocity is equal to $340 \mathrm{~km} \mathrm{~s}^{-1}$ at the photospheric radius $r=1$. The emission comes from $5<r<9$, in photospheric radius unit.

The permitted and forbidden $F W H M$ line components can be understood if we assume that the permitted lines are formed in the inner parts of the disk, while the forbiden ones issue from the external parts of the Keplerian disk.

Figure 15 shows the profile corresponding to an optically thin Keplerian disk inclined $12^{\circ}$ on the sky plane. The velocity at the photospheric radius $r=1$ is equal to $340 \mathrm{~km} \mathrm{~s}^{-1}$. The emission comes from the inner part $(5<r<9$, in photospheric 


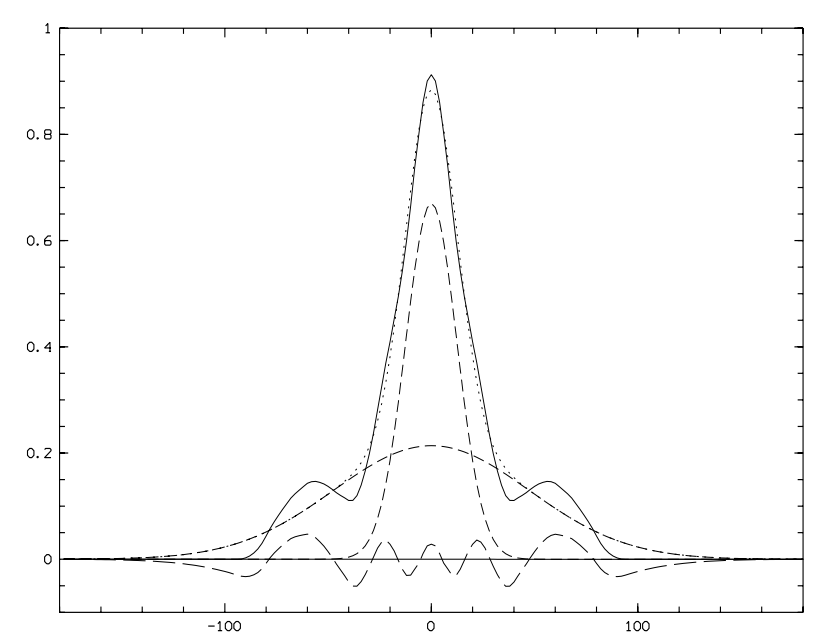

Fig. 16. Theoretical profile corresponding to the histogram of the emitting material radial velocities of an optically thin Keplerian disk inclined $12^{\circ}$ on the sky plane. An accreting wind generates the broad component. The component FWHM are the ones derived in Sect. 2.2. The Keplerian velocity is equal to $340 \mathrm{~km} \mathrm{~s}^{-1}$ at the photospheric radius $r=1$. The emission comes from $15<r<20$, in photospheric radius unit.

radius unit) of the optically thin disk whose total opening angle is $24^{\circ}$. The components flux ratio narrow/broad is close to 1 (the narrow component is slightly more intense) as was found for HD 45677, while the components FWHM are similar (44 and $194 \mathrm{~km} \mathrm{~s}^{-1}$ ) to the $\mathrm{Fe}$ II permitted lines ones.

Figure 16 shows the profile corresponding to the same disk, but with emission coming from outer regions $(15<r<20$, in photospheric radius unit). The component flux ratio narrow/broad is slightly higher than in Fig. 15, as it is the case for [Fe II] with regard to Fe II lines in HD 45677, while the components $F W H M$ are similar (28 and $\left.108 \mathrm{~km} \mathrm{~s}^{-1}\right)$ to the [Fe II] lines ones.

These two profiles model fairly well the mean component parameters we measured in HD 45677 as well for the permitted as for the forbidden $\mathrm{Fe}$ II lines.

\section{Discussion}

\subsection{Level population}

The results of our analysis concerning level population are summarised in Fig. 17 where we plot for the three groups of lines - forbidden, low excitation and high excitation permitted Fe II narrow components emission lines - the logarithm of the normalised line flux as a function of the excitation potential $\chi_{\mathrm{u}}$ of the upper level of the transitions for our 1999 data. The fluxes of the intermediate excitation permitted lines are corrected for the self-absorption as discussed above. The diagram is used to investigate the formation region of the three line groups. One normally expects the upper levels of the permitted lines to be de-populated with respect to the metastable levels. If no de-population occurs, the population laws for the upper levels and the lower levels should join. In Fig. 17, the mean population laws for the upper $(3150 \mathrm{~K})$ and the lower $(3900 \mathrm{~K})$ cross at the mean excitation potential value of $4.72 \mathrm{eV}$, to take

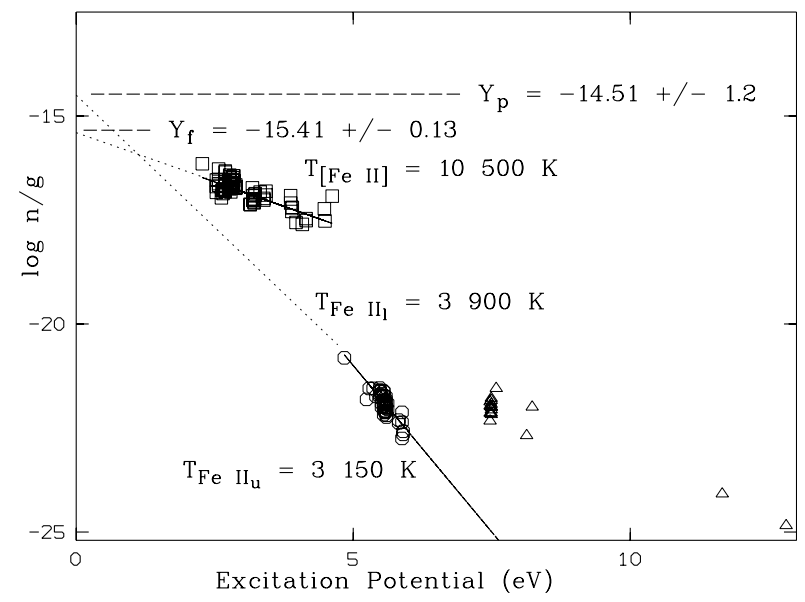

Fig. 17. Relative population of the $\mathrm{Fe}^{+}$levels in the [Fe II] (squares), and in the low and high excitation Fe II emission regions derived from the narrow emission components measured in the 1999 spectrum of HD 45677. The fluxes of the lower excitation emission lines (open circles) have been corrected for self-absorption, as discussed in the text. The high excitation emission lines are drawn as triangles. The straight lines represent the mean excitation temperatures of the upper levels of the [Fe II] and of the lower and upper levels of the Fe II emission lines, respectively. The crossing at $4.72 \mathrm{eV}$ marks the connection between the lower (3900 K) and upper (3150 K) Fe II level population laws. The zero points $Y(0 \mathrm{eV})$ are the estimated ground level relative populations.

into account the overall deviation of the population of the upper (odd) levels of the Fe II transitions with respect to the population of the lower (even) ones (see Sect. 3.1). The extrapolated ordinate value at $0 \mathrm{eV}$ of this law, given by Eq. (4), for the Fe II lines $(-14.51 \pm 1.2)$ turns out to be close to the value for the $[\mathrm{Fe}$ II] lines $(-15.41 \pm 0.13)$. This will be discussed in Sect. 5.2.

We get in this diagram a limit to the position of the metastable levels in the permitted line formation region. As forbidden and low excitation Fe II transitions have the metastable levels in common, we may note that the [Fe II] transition upper levels in the Fe II permitted lines region appear to lie in this diagram aproximately 2 to 3 dex under the location of the [Fe II] observed line ones. Consequently [Fe II] lines in the permitted line formation region cannot be observed.

Finally, the high excitation $\mathrm{Fe}^{+}$levels are clearly overpopulated with respect to the upper levels of the lower excitation Fe II emission lines. We attribute this overpopulation to the same fluorescence mechanism acting in other peculiar stars, such as KQ Pup, $\eta$ Car, and RR Tel (Muratorio et al. 1992; Viotti et al. 1999; Hartman \& Johansson 2000) whose optical and UV spectrum shows prominent $\mathrm{Fe}$ II lines arising from high excitation levels. Like in these cases, the far-UV radiation is most probably an important process of population of the $\mathrm{Fe}^{+}$ upper levels in the envelope of HD 45677, while probably dielectronic recombinations are unimportant.

\subsection{Column density}

From the position of the bend in the SAC plots of Fe II lines (Figs. 7 and 8 for 1999 data) we are able to determine the value 
Table 12. logarithm of the minimum $\mathrm{Fe}^{+}$column density $\frac{N_{\mathrm{c}}}{g_{\mathrm{c}}}$ (for an excitation potential $\chi_{\mathrm{c}}=2.75 \mathrm{eV}$ ) of both components emission line region at the different epochs studied. The first line display $(\mathrm{N})$ the narrow component region values, while the second line displays (B) the broad component region ones.

\begin{tabular}{cccc}
\hline \hline year & 1995 & 1999 & 2001 \\
\hline $\log \frac{N_{\mathrm{c}}}{g_{\mathrm{c}}}(\mathrm{N})$ & $14.6 \pm 0.1$ & $14.9 \pm 0.1$ & $14.73 \pm 0.1$ \\
$\log \frac{N_{\mathrm{c}}}{g_{\mathrm{c}}}(\mathrm{B})$ & $14.4 \pm 0.1$ & $14.5 \pm 0.1$ & $14.5 \pm 0.1$ \\
\hline
\end{tabular}

of $X_{\mathrm{c}}$ and hence through Eq. (3) the values of the $\mathrm{Fe}^{+}$column density of the $\mathrm{Fe}$ II permitted lines emitting regions for each studied epoch.

To derive the Fe II column density $\frac{N_{\mathrm{c}}}{g_{\mathrm{c}}}$, defined by Eq. (3), in the narrow component $\mathrm{Fe}$ II permitted line region, we have to estimate the velocity broadening of the opacity profile $v_{\mathrm{c}}$ in Eq. (3). The narrow components of the emission lines of HD 45677 should be formed in a region with a small velocity gradient in the line of sight, as discussed in Sect. 4, it is supposed to be an equatorial disk seen nearly pole on. If we are observing a disk in rotation, without being in its plane, the appropriate $v_{\mathrm{c}}$ to use, to which the line broadening along a line of sight is linked, is twice the thermal broadening velocity that has been derived from the [ $\mathrm{Fe}$ II] emission lines, as discussed in Sect. 3.3. The local velocity dispersion for a temperature $T_{\text {th }}=11500 \mathrm{~K}$ is given by $v_{\text {th }}=0.215 \times 10^{5} \sqrt{T_{\text {th }} / \mathrm{PA}}$ where $\mathrm{PA}=55.85$ for $\mathrm{Fe}^{+}$(Baratta et al. 1998). For the narrow components $v_{\mathrm{c}}=6.2 \mathrm{~km} \mathrm{~s}^{-1}$. As for the broad components, the appropriate $v_{\mathrm{c}}$ to use is given by our model, as the mean velocity along a line of sight, $v_{\mathrm{c}}$ is equal to $7 \mathrm{~km} \mathrm{~s}^{-1}$.

The different epochs and region $\log \frac{N_{\mathrm{c}}}{g_{\mathrm{c}}}$ values at $\chi_{\mathrm{c}}=$ $2.75 \mathrm{eV}$ are given in Table 12. If we extrapolate this column density to $0 \mathrm{eV}$ using 1.28 as the exponent of the power law of the lower terms population we obtain $\log \bar{N}\left(\mathrm{Fe}^{+}\right)=46.5$ in 1999. It is not certain that assumption (d), in Sect. 3, about the same Boltzmann population distribution for all metastable levels is correct for the more excited of these levels (see Verner et al. 2002). Therefore, if we are very conservative and suppose that the mean excitation temperature down to $0 \mathrm{ev}$ is $10000 \mathrm{~K}$, typical of HII regions, as well as being of the order of what we find here for the less excited forbidden [Fe II] lines with lower levels at or near $0 \mathrm{ev}$, the column density values might be smaller by a factor of about 2.1 dex.

The different narrow and broad component column densities in Table 12 are very similar. The eventual column density variations with time are of the order of the precision.

As for forbidden lines, Fig. 10 shows a marginally significant bending of the data points towards large optical thicknesses, as if the strongest [Fe II] lines were slightly selfabsorbed. The curve bend, if real, would correspond in this figure to a self absorption factor of 3 for strongest of the observed forbidden lines, much less than what is observed for the strongest permitted lines, as the optical depth range is lower for forbidden lines. It would correspond to an $\mathrm{Fe}^{+}$column density about 100 times larger than that above derived for the permitted lines, which seems to us unrealistic.
Table 13. Emission line region minimum sizes at the different epochs studied (in $\mathrm{cm}$ ). The first line display the narrow component region values $(\mathrm{N})$, while the second line displays the broad component region ones (B).

\begin{tabular}{cccc}
\hline \hline year & 1995 & 1999 & 2001 \\
\hline $\log R^{\prime}(\mathrm{N})$ & $13.1 \pm 0.5$ & $12.9 \pm 0.3$ & $12.5 \pm 0.4$ \\
$\log R^{\prime}(\mathrm{B})$ & $12.8 \pm 0.8$ & $13.0 \pm 0.4$ & $12.6 \pm 0.5$ \\
\hline
\end{tabular}

The vertical overlapping of the multiplets provides the zero point of the ordinates: $Y_{f}=-15.41 \pm 0.13$ (see Table 9). This quantity is related to the total number $\bar{N}\left(\mathrm{Fe}^{+}\right)$of $\mathrm{Fe}^{+}$ions in the $[\mathrm{Fe}$ II] emitting volume through the relationship (see Baratta et al. 1998):

$\log \bar{N}\left(\mathrm{Fe}^{+}\right) / d^{2}=Y_{f}+\log U+16.977$,

where $U$ is the partition function of $\mathrm{Fe}^{+}$.

Assuming for HD 45677 a distance of 420 pc (Zorec 1998), we obtain $\log \bar{N}\left(\mathrm{Fe}^{+}\right)=45.6 \pm 0.13$ which gives in 1999 , for a cosmic abundance, a total hydrogen mass of $1.7 \times 10^{26} \mathrm{~g}$, with a precision factor estimated as 0.13 .

Notice that the $\log \bar{N}\left(\mathrm{Fe}^{+}\right)=46.5 \pm 1.3$ value of the Fe II permitted emitting region, obtained with a value of 1.28 for the lower level constant $p$ of Eq. (1), is close to the forbidden Fe II region one.

\subsection{Size of the emitting region}

From the fit results summarised in Tables 6 and 7 we can calculate, using Eqs. (3) and (4), the apparent size of both emitting line regions.

For 1999 we obtain $\log R^{\prime}=12.90 \pm 0.34$ for the narrow line region. The values of $\log R^{\prime}$ in Table 13 are typical radii of the line emitting region, equal to $\sqrt{\frac{S \cos i}{\pi}}$. The radius of a B2 V star is according to Drilling \& Landolt (2000 in "Astrophysical Quantities", p. 389) near $4 \times 10^{11} \mathrm{~cm}$. The size of the region where the narrow Fe II emisison lines originate, probably a disk, turns out to be 20 (from 9 to 44) times that of the stellar radius. The model we presented in Sect. 4 is consistent with these results as the permitted line profile similar to the one observed is emitted from a region whose surface is that of a disk of 7 stellar radii. The assumption has been made in the $R^{\prime}$ determination that the population laws for the upper levels and the lower levels join at $4.72 \mathrm{eV}$. Their values obtained are therefore minima, corresponding to maximum $\mathrm{w}$ values.

The $\log R^{\prime}$ values obtained from 1995 and 2001 data in Table 13 remain close to the limits evaluated from the 1999 data in Table 13.

As for the broad component emitting region apparent surface, the values of $\log R^{\prime}$ are very close, at least in 1999 to the narrow component one. This result is consistent with the model of a disk seen nearly pole-on, developped in Sect. 4, as the regions in which each component is emitted, being seen under the same opening angle, are supposed to have similar apparent surfaces. 
As for the narrow component $\mathrm{Cr}$ II emitting region, we used $v_{0}=6 \mathrm{~km} \mathrm{~s}^{-1}$ (twice the $10000 \mathrm{~K}$ thermal broadening velocity) and the values of Table 11, hence a Cr II column density $\frac{N_{0}}{g_{0}}=$ $15.98 \pm 0.6$. The $\log R^{\prime}$ value derived in $1999(12.2 \pm 0.8)$ is only slightly smaller than that of the $\mathrm{Fe}$ II emitting regions.

\section{Conclusions}

The SAC method has given consistent results. We have obtained the properties of what is probably a disk of HD 45677 , including limits on a characteristic column density and a characteristic radius of the $\mathrm{Fe}$ II emitting region. These estimates require reasonable assumptions, one of which is a true Boltzmann distribution of the populations of the lower even metastable levels and another that the Boltzmann-like law of the populations of the odd upper levels of strong optical lines are not more than what would be expected from a smooth fit between these odd and even population laws. We have demonstrated that the well known split emission line profile is in fact due to absorption components like in the ultraviolet. We have demonstrated that emission lines have narrow and broad components. We have shown that the double emission profile can be explained with a disk plus a flow (perhaps a wind) moving in or out of the disk. The Fe II forbidden lines show a similar two component structure, but the smaller width of each component is consistent with their production in a more external part of the disk, where the Keplerian velocity is lower. The examination of the fluxes of the narrow [Fe II] components has shown that the metastable $\mathrm{Fe}^{+}$levels follow a Boltzmann-type law with a mean excitation temperature around 11000 K. Such a value may actually represent the electron temperature of the [Fe II] emitting region, if the metastable levels are in equilibrium with the ground term through electron collisions. This is probably the case of HD 45677, since we expect the electron density to be large enough $\left(N_{\mathrm{e}} \geq 10^{6} \mathrm{~cm}^{-3}\right)$ for bringing the metastable levels to LTE (Nussbaumer \& Storey 1988). The low electron temperature with respect to the stellar surface temperature may be due to cooling of the $[\mathrm{Fe}$ II] region by the large line emission of this ion. Indeed, low electron temperatures were predicted by Verner et al. (1999) and Verner et al. (2002) for the $\eta$ Car Weigelt blobs that are strong $\mathrm{Fe}^{+}$emitters. Fairly low [Fe II] excitation temperatures were derived for $\eta$ Car (12000 K, Viotti et al. 1999) and for the symbiotic star RR Tel (6600 K Kotnik-Karuza et al. 2002).

The narrow components of the Fe II permitted emission lines in HD 45677 are slightly, but significantly broader than the corresponding [Fe II] components. From the SAC analysis of the $\mathrm{Fe}$ II emission lines we have derived an $\mathrm{Fe}^{+}$lower level excitation temperature much smaller than that derived from the [Fe II] lines, namely around $3900 \mathrm{~K}$. We take these results as evidence of different (but possibly partially overlapping) emitting regions. The derived excitation temperatures of the lower and upper levels of the permitted Fe II lines are much smaller than that expected if $\mathrm{Fe}^{+}$were in thermodynamical equilibrium with the local plasma. Verner et al. (2002) calculated the formation of singly ionized iron in the Weigelt blobs of $\eta$ Car. According to them $\mathrm{Fe}^{+}$emission is the most important coolant in the blobs. Fe II emission originates at electron temperatures
$5000 \mathrm{~K} \leq T_{\mathrm{e}} \leq 7500 \mathrm{~K}$. This can also be seen by comparing their Figs. 8 and 9. We argue that for HD 45677 the upper levels of the permitted low excitation transitions are mostly populated by the diluted stellar UV radiation followed by spontaneous decay, and that in the Fe II region the radiation density is high enough to make the radiation induced transitions more important than electron collisions. Similar considerations can be made for $\mathrm{Fe}$ II and [Fe II] broad components, as their emitting region parameters appear fairly close to the narrow components ones (see Tables 6 and 7 for Fe II lines and Tables 9 and 10 for [Fe II] lines).

The Fe II metastable level, as well as the lower level (Table 6) excitation temperatures are suspected to increase with time. The high excitation anomalous multiplets may indicate the presence of inhomogeneities.

The shape of the iron broad component is insufficiently known now to decide about the structure of the non-disk component. Nevertheless the similarity of the physical parameters derived for narrow and broad components $\left(T_{\text {exc }}, \log \frac{N_{\mathrm{O}}}{g_{0}}\right)$ suggest that both emitting regions, if not the same (different $F W H M)$ are physically linked.

Our identification of the broad components, and the suspected variability of both emitting regions physical parameters should stimulate in future new higher quality observations (especially in the blue) in order to study more precisely the shape of the emission lines.

Acknowledgements. We are grateful to R. F. Viotti for very constructive suggestions. This work is partly based on contract of the Consiglio Nazionale delle Ricerche (CNR).

\section{References}

Allen, D. A., \& Swings, J. P. 1976, A\&A, 47, 293

Allen's Astrophysical Quantities Fourth edition, 2000, ed. A. N. Cox

Andrillat, Y., Jaschek, C., \& Jaschek, M. 1997, A\&AS, 124, 441

Baratta, G. B., Friedjung, M., Muratorio, G., Ross, C., \& Viotti, R. 1998, The Self Absorption Curve Method, A Users' Munual, IAS Internal Report, December 1998

Cidale, L., Zorec, J., \& Tringaniello, L. 2001, A\&A, 368, 160

Coyne, G. V., \& Vrba, F. J. 1976, ApJ, 207, 790

Eriksson, M., Johansson, S., \& Wahlgren, G. M. 2004, A\&A, 422, 987

Friedjung, M., \& Muratorio, G. 1987, A\&A, 188, 100

Grady, C. A., Bjorkman, K. S., Shepherd, D., et al. 1993, ApJ, 415, L39

Hartman, H., \& Johansson, S. 2000, A\&A, 359, 627

Israelian, G., \& Musaev, F. 1997, A\&A, 328, 339

Israelian, G., Friedjung, M., Graham, J., et al. 1996, A\&A, 311, 643

Kastner, S. O. 1999, A\&A, 351, 1016

Kotnik-Karuza, D., Friedjung, M., \& Selvelli, P. L. 2002, A\&A, 381, 507

Kurucz, R. L. 1979, ApJS, 40, 1

Kurucz, R. L. 1991, Precision photometry: Astrophysics of the galaxy, in ed. A. G. Davis Philip, A. R. Upgren, \& K. A. James (Schenectady, New York: L. Davis press)

Kurucz, R. L. 1993, SAO KURUCZ CD-ROM, No. 18

Lamers, H. J. G. L. M., Zickgraf, F. J., de Winter, D., Houziaux, L., \& Zorec, J. 1998, A\&A, 340, 117

Muratorio, G., \& Friedjung, M. 1988, A\&A, 190, 103

Muratorio, G., Viotti, R., Friedjung, M., Baratta, G. B., \& Rossi, C. 1992, A\&A, 258, 423 
Moore, Ch. 1945, A multiplet table of astrophysical interest, NSRDSNBS 40 I ed. 1945 and successive editions

Nussbaumer, H., \& Storey, P. J. 1998, A\&A, 193, 327

Quinet, P., Le Dourneuf, M., \& Zeippen, C. J. 1996, A\&AS, 120, 361

Schulte-Ladbeck, R. E., Shepherd, D. S., Nordsieck, K. H., et al. 1992, ApJ, 401, L105

Sitko, M. L., Grady, C. A., Hanner, M. S., Lynch, D. K., \& Russell, R. W. 1994, in Circumstellar Dust Disks and Planet Formation, ed. R. Ferlet, \& A. Vidal-Madjar (Paris: Éditions Frontières), 389

Swings, J. P. 1973, A\&A, 26, 443

van den Ancker, M. E., Blondel, P. F., Tjin, A Djie, H. R. E., et al. 2004, MNRAS, 349, 1516

Verner, E. M., Verner, D. A., Korista, K. T., et al. 1999, ApJS, 120, 101

Verner, E. M., Gull, T. R., Bruhweiler, F., et al. 2002, ApJ, 581, 1154
Viotti, R. 1976, ApJ, 204, 293

Viotti, R., Rossi, C., \& Baratta, G. B. 1999, in Eta Carinae at the Millenium, ed. J. Morse, R. Humphreys, \& A. Daminelli, ASP Conf. Ser., 179, 184

Viotti, R. F., Spector, N., Baratta, G. B., \& Rossi, C. 2000, A\&A, 363, 343

de Winter, D., \& van den Ancker, M. E. 1997, A\&AS, 121, 275

Zickgraf, F. J. 1998, in Dusty B[e] stars, ed. A. M. Hubert, \& C. Jaschek

Zickgraf, F. J. 2001, A\&A, 375, 122

Zickgraf, F. J. 2003, A\&A, 408, 257

Zorec, J. 1998, in B[e] stars, ed. A. M. Hubert, \& C. Jaschek (Kluwer Ac. Pub.), 27

Zorec, J., Moujtahid, A., Ballereau, D., \& Chauville, J. 1998, in B[e] stars, ed. A. M. Hubert, \& C. Jaschek (Kluwer Ac. Pub.), 55 
G. Muratorio et al.: Analysis of the circumstellar environment of the B[e] star HD 45677 (FS Canis Majoris), Online Material $p 1$

\section{Online Material}


G. Muratorio et al.: Analysis of the circumstellar environment of the B[e] star HD 45677 (FS Canis Majoris), Online Material p 2

Table 1. Log of the AURELIE observations of HD 45677. UT is the mid-exposure time. Exposure time is given in seconds and spectral range in $\AA$. $S / N$ ratio refers to the continuum.

\begin{tabular}{ccccrr}
\hline \hline Date & UT & exp & sp range & $R$ & $S / N$ \\
\hline 1995 Jan. 11 & $23: 16$ & 7200 & $4080-4480$ & 7000 & 200 \\
1995 Jan. 12 & $0: 40$ & 7200 & $4900-5320$ & 8000 & 150 \\
1995 Jan. 13 & $23: 20$ & 7200 & $5700-6550$ & 5000 & 400 \\
1995 Jan. 14 & $23: 00$ & 7200 & $4480-4900$ & 7000 & 300 \\
1995 Jan. 16 & $23: 20$ & 4000 & $5340-5760$ & 9000 & 350 \\
1995 Jan. 17 & $0: 45$ & 3200 & $4900-5320$ & 8000 & 100 \\
1999 Feb. 02 & $21: 50$ & 8100 & $4455-4660$ & 15000 & 100 \\
1999 Feb. 03 & $20: 50$ & 8100 & $5155-5360$ & 15000 & 150 \\
1999 Feb. 04 & $21: 20$ & 2700 & $4640-5060$ & 8000 & 70 \\
1999 Feb. 05 & $20: 25$ & 8100 & $4060-4500$ & 7000 & 70 \\
1999 Feb. 06 & $20: 30$ & 8100 & $6140-6550$ & 9000 & 140 \\
1999 Feb. 06 & $22: 40$ & 5400 & $5320-5740$ & 11000 & 150 \\
1999 Feb. 07 & $20: 00$ & 5400 & $5730-6160$ & 10000 & 170 \\
1999 Feb. 07 & $22: 10$ & 5400 & $5320-5740$ & 9000 & 150 \\
2001 Dec. 06 & $1: 00$ & 3600 & $4390-4610$ & 15000 & 200 \\
2001 Dec. 06 & $2: 40$ & 4500 & $5150-5360$ & 18000 & 200 \\
2001 Dec. 07 & $2: 40$ & 1800 & $6100-6310$ & 20000 & 150 \\
2002 Jan. 16 & $21: 45$ & 3600 & $6310-6520$ & 21000 & 200 \\
2002 Jan. 16 & $23: 00$ & 3600 & $6100-6310$ & 20000 & 150 \\
2002 Jan. 17 & $0: 20$ & 3600 & $5800-6010$ & 19000 & 300 \\
\hline
\end{tabular}


Table 2. Identification, intensity and radial velocity of the line components measured in the 1995, 1999 and 2001-2002 AURELIE spectra of HD 45677. For each line, the successive rows give the narrow and broad emissions, and the central absorption, when present. The successive columns give for each line component the rest wavelength, the atomic specie and multiplet number, the flux in $10^{-13} \mathrm{erg} \mathrm{cm}^{-2} \mathrm{~s}^{-1}, F W H M$ in $\AA$, and heliocentric radial velocity in $\mathrm{km} \mathrm{s}^{-1}$ units (for each year) in the three observation epochs. For the absorption components intensities are given as negative fluxes.

\begin{tabular}{|c|c|c|c|c|c|c|c|c|c|c|c|}
\hline IDENT & & & $\overline{\text { F95 }}$ & W95 & V95 & F99 & W99 & V99 & F01 & W01 & V01 \\
\hline 4068.62 & S II & $1 \mathrm{~F}$ & 9.86 & $\overline{0.8}$ & 29 & 10.38 & 0.8 & 18 & & & \\
\hline 4076.22 & S II & $1 \mathrm{~F}$ & 1.71 & 0.7 & 42 & 1.51 & 0.6 & 31 & & & \\
\hline 4101.737 & $\mathrm{H} \delta$ & & -24 & 1.6 & -53 & -14.0 & 1.6 & -44 & & & \\
\hline 4101.737 & $\mathrm{H} \delta$ & & -84 . & 17. & 24 & -90.8 & 17. & 23 & & & \\
\hline 4101.737 & $\mathrm{H} \delta$ & & 35. & 3.1 & 24 & 33.4 & 3.0 & 23 & & & \\
\hline 4101.737 & $\mathrm{H} \delta$ & & -12 & 1.0 & 29 & -13.0 & 1.0 & 29 & & & \\
\hline 4114.48 & Fe II & $23 \mathrm{~F}$ & 0.85 & 0.6 & 31 & 0.89 & 0.6 & 20 & & & \\
\hline 4114.48 & Fe II & $23 \mathrm{~F}$ & 0.40 & 1.6 & 31 & 0.97 & 1.3 & 20 & & & \\
\hline 4120.991 & $\mathrm{He} \mathrm{I}$ & 16 & -3.41 & 1.6 & 17 & -3.51 & 1.7 & 12 & & & \\
\hline 4128.054 & Si II & 3 & -2.43 & 1.4 & 8 & -2.28 & 1.0 & 26 & & & \\
\hline 4128.748 & Fe II & 27 & 0.18 & 0.9 & 23 & 0.18 & 0.9 & 24 & & & \\
\hline 4128.748 & Fe II & 27 & 0.15 & 2.5 & 23 & 0.19 & 2.5 & 24 & & & \\
\hline 4128.748 & Fe II & 27 & -0.04 & 0.7 & 26 & -0.04 & 0.7 & 28 & & & \\
\hline 4130.894 & Si II & 3 & -2.31 & 1.4 & 12 & -2.38 & 1.0 & 25 & & & \\
\hline 4143.761 & $\mathrm{He} \mathrm{I}$ & 53 & -10.5 & 3.3 & 25 & -10.6 & 3.6 & 23 & & & \\
\hline 4173.461 & Fe II & 27 & 1.78 & 0.9 & 27 & 2.45 & 0.9 & 40 & & & \\
\hline 4173.461 & Fe II & 27 & 1.28 & 2.5 & 27 & 2.48 & 2.5 & 40 & & & \\
\hline 4173.461 & Fe II & 27 & -0.83 & 0.7 & 29 & -1.20 & 0.5 & 45 & & & \\
\hline 4177.21 & Fe II & $21 \mathrm{~F}$ & 1.32 & 0.7 & 27 & 1.54 & 0.7 & 26 & & & \\
\hline 4177.21 & Fe II & $21 \mathrm{~F}$ & 0.36 & 1.6 & 27 & 0.80 & 1.5 & 26 & & & \\
\hline 4178.862 & Fe II & 28 & 2.50 & 0.9 & 26 & 3.12 & 0.9 & 18 & & & \\
\hline 4178.862 & Fe II & 28 & 2.35 & 2.5 & 26 & 2.48 & 2.5 & 18 & & & \\
\hline 4178.862 & Fe II & 28 & -0.25 & 0.5 & 29 & -0.12 & 0.5 & 21 & & & \\
\hline 4179.421 & Cr II & 26 & 0.31 & 0.9 & 27 & 0.27 & 0.9 & 26 & & & \\
\hline 4179.421 & Cr II & 26 & 0.10 & 2.5 & 27 & 0.62 & 2.5 & 26 & & & \\
\hline 4179.421 & Cr II & 26 & -0.12 & 0.5 & 30 & -0.11 & 0.5 & 30 & & & \\
\hline 4201.74 & Ni II & $3 F$ & 0.94 & 0.6 & -14 & 1.14 & 0.8 & -1 & & & \\
\hline 4205.473 & Fe II & 22 & 0.25 & 0.6 & 22 & 0.27 & 0.6 & 23 & & & \\
\hline 4211.099 & Fe II & $23 \mathrm{~F}$ & 0.35 & 0.6 & 23 & 0.45 & 0.6 & 12 & & & \\
\hline 4231.56 & Fe II & $21 \mathrm{~F}$ & 0.17 & 0.7 & 28 & 0.36 & 0.7 & 26 & & & \\
\hline 4233.172 & Fe II & 27 & 5.11 & 0.9 & 28 & 4.15 & 0.9 & 25 & & & \\
\hline 4233.172 & $\mathrm{Fe}$ II & 27 & 6.19 & 2.5 & 28 & 9.66 & 2.5 & 25 & & & \\
\hline 4233.172 & Fe II & 27 & -1.07 & 0.6 & 32 & -0.83 & 0.6 & 32 & & & \\
\hline 4242.364 & Cr II & 31 & & & & 0.60 & 0.7 & 18 & & & \\
\hline 4242.364 & Cr II & 31 & & & & 0.80 & 2.7 & 18 & & & \\
\hline 4242.364 & Cr II & 31 & & & & -0.59 & 0.4 & 20 & & & \\
\hline 4243.98 & Fe II & $21 \mathrm{~F}$ & 5.45 & 0.7 & 25 & 6.39 & 0.7 & 22 & & & \\
\hline 4243.98 & Fe II & $21 \mathrm{~F}$ & 2.56 & 1.6 & 23 & 1.80 & 1.4 & 22 & & & \\
\hline 4244.81 & Fe II & $21 \mathrm{~F}$ & 1.48 & 0.7 & 27 & 1.37 & 0.7 & 24 & & & \\
\hline 4244.81 & Fe II & $21 \mathrm{~F}$ & 0.50 & 1.6 & 27 & 1.15 & 1.4 & 24 & & & \\
\hline 4267.259 & C II & & -2.21 & 1.5 & 28 & -2.66 & 1.8 & 24 & & & \\
\hline 4276.83 & Fe II & $21 \mathrm{~F}$ & 4.28 & 0.7 & 25 & 4.78 & 0.8 & 22 & & & \\
\hline 4276.83 & Fe II & $21 \mathrm{~F}$ & 1.29 & 1.6 & 25 & 1.25 & 1.4 & 22 & & & \\
\hline 4287.4 & Fe II & $7 F$ & 10.28 & 0.7 & 24 & 10.46 & 0.7 & 21 & & & \\
\hline 4287.4 & Fe II & $7 F$ & 3.37 & 1.6 & 24 & 4.01 & 1.4 & 22 & & & \\
\hline 4296.572 & Fe II & 28 & 1.48 & 0.9 & 31 & 1.61 & 0.9 & 24 & & & \\
\hline 4296.572 & $\mathrm{Fe}$ II & 28 & 0.56 & 2.5 & 31 & 1.36 & 2.5 & 24 & & & \\
\hline 4296.572 & Fe II & 28 & -0.63 & 0.7 & 32 & -0.72 & 0.5 & 25 & & & \\
\hline 4303.176 & Fe II & 27 & 1.16 & 0.9 & 30 & 1.35 & 0.9 & 23 & & & \\
\hline 4303.176 & Fe II & 27 & 0.95 & 2.5 & 30 & 1.49 & 2.5 & 23 & & & \\
\hline 4303.176 & Fe II & 27 & -0.39 & 0.6 & 32 & -0.62 & 0.6 & 28 & & & \\
\hline 4305.9 & Fe II & $21 \mathrm{~F}$ & 1.35 & 0.7 & 24 & 1.77 & 0.7 & 20 & & & \\
\hline 4305.9 & Fe II & $21 \mathrm{~F}$ & 0.48 & 1.6 & 24 & 0.35 & 1.4 & 20 & & & \\
\hline
\end{tabular}


G. Muratorio et al.: Analysis of the circumstellar environment of the B[e] star HD 45677 (FS Canis Majoris), Online Material p 4

Table 2. continued.

\begin{tabular}{|c|c|c|c|c|c|c|c|c|c|c|c|}
\hline$\overline{\text { IDENT }}$ & & & 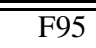 & "W95 & V95 & $\overline{\text { F99 }}$ & 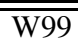 & "V99 & $\overline{\overline{\text { F01 }}}$ & $\overline{\mathrm{W} \text { W01 }}$ & $\overline{\mathrm{V} \text { V01 }}$ \\
\hline 4314.31 & $\mathrm{Fe}$ II & 32 & 0.31 & 0.8 & 35 & 0.85 & 0.9 & 41 & & & \\
\hline 4319.62 & $\mathrm{Fe}$ II & $21 \mathrm{~F}$ & 3.06 & 0.7 & 24 & 3.25 & 0.7 & 22 & & & \\
\hline 4319.62 & $\mathrm{Fe} I I$ & $21 \mathrm{~F}$ & 0.85 & 1.6 & 25 & 0.87 & 1.4 & 22 & & & \\
\hline 4326.85 & Ni II & $3 \mathrm{~F}$ & 1.34 & 0.8 & -17 & 1.40 & 0.7 & -18 & & & \\
\hline 4340.468 & $\mathrm{H} \gamma$ & & -33.2 & 1.6 & -54 & -23.6 & 1.6 & -43 & & & \\
\hline 4340.468 & $\mathrm{H} \gamma$ & & -58.9 & 17.0 & 24 & -57.2 & 17.0 & 24 & & & \\
\hline 4340.468 & $\mathrm{H} \gamma$ & & 84.6 & 3.1 & 24 & 73.7 & 3.0 & 24 & & & \\
\hline 4340.468 & $\mathrm{H} \gamma$ & & -22.6 & 1.0 & 29 & -22.6 & 1.0 & 28 & & & \\
\hline 4346.85 & $\mathrm{Fe}$ II & $21 \mathrm{~F}$ & 1.19 & 0.7 & 26 & 1.32 & 0.7 & 23 & & & \\
\hline 4346.85 & $\mathrm{Fe}$ II & $21 \mathrm{~F}$ & 0.40 & 1.6 & 26 & 0.42 & 1.5 & 23 & & & \\
\hline 4351.769 & $\mathrm{Fe} I I$ & 27 & 3.04 & 0.9 & 23 & 2.43 & 0.9 & 23 & & & \\
\hline 4351.769 & $\mathrm{Fe}$ II & 27 & 2.23 & 2.5 & 23 & 3.53 & 2.5 & 23 & & & \\
\hline 4351.769 & $\mathrm{Fe}$ II & 27 & -0.94 & 0.7 & 26 & -0.85 & 0.6 & 29 & & & \\
\hline 4352.78 & $\mathrm{Fe}$ II & $21 \mathrm{~F}$ & 2.01 & 0.7 & 21 & 2.19 & 0.7 & 22 & & & \\
\hline 4352.78 & $\mathrm{Fe}$ II & $21 \mathrm{~F}$ & 0.95 & 1.6 & 21 & 0.69 & 1.4 & 22 & & & \\
\hline 4358.37 & $\mathrm{Fe}$ II & $21 \mathrm{~F}$ & 3.42 & 0.7 & 23 & 3.08 & 0.7 & 17 & & & \\
\hline 4358.37 & $\mathrm{Fe}$ II & $21 \mathrm{~F}$ & 1.19 & 1.6 & 23 & 1.58 & 1.4 & 17 & & & \\
\hline 4359.34 & $\mathrm{Fe}$ II & $7 F$ & 7.64 & 0.7 & 23 & 7.14 & 0.7 & 21 & & & \\
\hline 4359.34 & $\mathrm{Fe}$ II & $7 F$ & 1.35 & 1.6 & 23 & 3.24 & 1.4 & 21 & & & \\
\hline 4372.43 & $\mathrm{Fe} I I$ & $21 \mathrm{~F}$ & 1.48 & 0.7 & 21 & 1.40 & 0.7 & 21 & & & \\
\hline 4372.43 & $\mathrm{Fe}$ II & $21 \mathrm{~F}$ & 0.43 & 1.6 & 21 & 0.69 & 1.4 & 21 & & & \\
\hline 4375.71 & CuII & $1 \mathrm{~F}$ & 0.66 & 0.8 & 19 & 0.92 & 0.8 & 23 & & & \\
\hline 4382.75 & $\mathrm{Fe}$ II & $6 \mathrm{~F}$ & 0.76 & 0.7 & 23 & 0.50 & 0.7 & 23 & & & \\
\hline 4382.75 & $\mathrm{Fe}$ II & $6 \mathrm{~F}$ & 0.24 & 1.6 & 23 & 0.26 & 1.6 & 23 & & & \\
\hline 4384.319 & $\mathrm{Fe}$ II & 32 & 0.58 & 0.9 & 23 & 0.94 & 0.9 & 18 & & & \\
\hline 4384.319 & $\mathrm{Fe}$ II & 32 & 0.99 & 2.5 & 23 & 1.61 & 2.5 & 18 & & & \\
\hline 4385.387 & $\mathrm{Fe}$ II & 27 & 0.89 & 0.9 & 26 & 1.11 & 0.9 & 29 & & & \\
\hline 4385.387 & $\mathrm{Fe} I I$ & 27 & 0.87 & 2.5 & 26 & 1.24 & 2.5 & 29 & & & \\
\hline 4385.387 & $\mathrm{Fe} I I$ & 27 & -0.30 & 0.6 & 29 & -0.78 & 0.6 & 36 & & & \\
\hline 4387.929 & $\mathrm{He} \mathrm{I}$ & 51 & -6.73 & 2.4 & 28 & -6.17 & 2.0 & 27 & & & \\
\hline 4413.601 & Fe II & 32 & 0.22 & 0.9 & 26 & 0.36 & 0.9 & 26 & 0.41 & 0.7 & 26 \\
\hline 4413.601 & $\mathrm{Fe}$ II & 32 & 0.27 & 2.7 & 26 & 0.13 & 2.7 & 26 & 0.26 & 2.7 & 26 \\
\hline 4413.601 & $\mathrm{Fe} I I$ & 32 & -0.27 & 0.6 & 28 & -0.15 & 0.6 & 27 & -0.66 & 0.5 & 27 \\
\hline 4413.78 & $\mathrm{Fe}$ II & $7 F$ & 5.95 & 0.8 & 22 & 5.26 & 0.7 & 24 & 5.33 & 0.5 & 20 \\
\hline 4413.78 & $\mathrm{Fe}$ II & $7 F$ & 2.22 & 1.6 & 22 & 2.60 & 1.5 & 24 & 1.09 & 1.6 & 20 \\
\hline 4416.27 & $\mathrm{Fe}$ II & $6 \mathrm{~F}$ & 5.42 & 0.8 & 24 & 5.43 & 0.8 & 23 & 4.50 & 0.5 & 20 \\
\hline 4416.27 & $\mathrm{Fe} I I$ & $6 \mathrm{~F}$ & 1.59 & 1.6 & 24 & 1.74 & 1.6 & 23 & 1.87 & 1.6 & 20 \\
\hline 4416.83 & Fe II & 27 & 1.07 & 0.9 & 24 & 2.35 & 0.9 & 22 & 1.50 & 0.7 & 25 \\
\hline 4416.83 & $\mathrm{Fe}$ II & 27 & 1.86 & 2.5 & 24 & 1.24 & 2.5 & 22 & 0.56 & 2.6 & 25 \\
\hline 4416.83 & $\mathrm{Fe} I I$ & 27 & -0.88 & 0.6 & 26 & -1.61 & 0.6 & 25 & -1.30 & 0.5 & 26 \\
\hline 4432.45 & $\mathrm{Fe}$ II & $6 \mathrm{~F}$ & 0.59 & 0.7 & 23 & 0.59 & 0.8 & 22 & 0.49 & 0.5 & 22 \\
\hline 4432.45 & $\mathrm{Fe}$ II & $6 \mathrm{~F}$ & 0.28 & 1.6 & 23 & 0.20 & 1.6 & 22 & 0.23 & 1.6 & 22 \\
\hline 4437.551 & $\mathrm{He} \mathrm{I}$ & 50 & -0.93 & 1.3 & 28 & -0.77 & 1.3 & 26 & & & \\
\hline 4452.11 & $\mathrm{Fe}$ II & $7 F$ & 3.25 & 0.7 & 21 & 3.86 & 0.7 & 22 & 3.02 & 0.5 & 20 \\
\hline 4452.11 & $\mathrm{Fe}$ II & $7 F$ & 1.35 & 1.6 & 23 & 1.38 & 1.4 & 22 & 0.78 & 1.6 & 20 \\
\hline 4457.95 & $\mathrm{Fe}$ II & $6 \mathrm{~F}$ & 2.91 & 0.7 & 22 & 2.82 & 0.6 & 24 & 2.63 & 0.5 & 20 \\
\hline 4457.95 & $\mathrm{Fe} I I$ & $6 \mathrm{~F}$ & 0.56 & 1.6 & 23 & 1.39 & 1.4 & 24 & 0.62 & 1.6 & 20 \\
\hline 4471.473 & $\mathrm{He} \mathrm{I}$ & 14 & -10.3 & 2.6 & 27 & -8.81 & 2.3 & 33 & & & \\
\hline 4474.91 & $\mathrm{Fe} I I$ & $7 F$ & 1.65 & 0.7 & 20 & 1.67 & 0.6 & 23 & 1.52 & 0.5 & 21 \\
\hline 4474.91 & $\mathrm{Fe}$ II & $7 F$ & 0.41 & 1.6 & 22 & 0.29 & 1.5 & 23 & 0.55 & 1.6 & 21 \\
\hline 4481.126 & Mg II & 4 & -12.0 & 2.3 & 16 & 11.49 & 4.1 & 28 & -11.1 & 2.0 & 40 \\
\hline 4481.126 & Mg II & 4 & 16.26 & 3.5 & 15 & -8.34 & 1.9 & 25 & 16.49 & 4.2 & 40 \\
\hline 4488.75 & $\mathrm{Fe} I \mathrm{II}$ & $6 \mathrm{~F}$ & 1.25 & 0.6 & 18 & 1.16 & 0.4 & 23 & 1.21 & 0.5 & 19 \\
\hline 4488.75 & $\mathrm{Fe}$ II & $6 \mathrm{~F}$ & 0.32 & 1.6 & 22 & 0.49 & 1.6 & 23 & 0.31 & 1.6 & 19 \\
\hline 4489.183 & $\mathrm{Fe}$ II & 37 & 0.89 & 0.9 & 25 & 0.91 & 0.9 & 24 & 0.70 & 0.7 & 25 \\
\hline 4489.183 & $\mathrm{Fe}$ II & 37 & 0.50 & 2.5 & 25 & 1.14 & 2.5 & 24 & 0.53 & 2.7 & 25 \\
\hline 4489.183 & $\mathrm{Fe}$ II & 37 & -0.14 & 0.6 & 29 & -0.14 & 0.8 & 27 & -0.24 & 0.4 & 28 \\
\hline
\end{tabular}


G. Muratorio et al.: Analysis of the circumstellar environment of the B[e] star HD 45677 (FS Canis Majoris), Online Material p 5

Table 2. continued.

\begin{tabular}{|c|c|c|c|c|c|c|c|c|c|c|c|}
\hline$\overline{\text { IDENT }}$ & & & $\overline{\text { F95 }}$ & $\overline{\text { W95 }}$ & 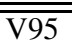 & $\overline{\text { F99 }}$ & $\overline{\text { W99 }}$ & "V99 & $\overline{\mathrm{F} \text { F01 }}$ & $\overline{\overline{\text { W01 }}}$ & $\overline{\overline{\text { V01 }}}$ \\
\hline 4491.405 & Fe II & 37 & 2.17 & 0.9 & 22 & 1.82 & 0.8 & 22 & 1.84 & 0.7 & 16 \\
\hline 4491.405 & Fe II & 37 & 1.05 & 2.5 & 22 & 0.84 & 2.6 & 22 & 1.84 & 2.7 & 16 \\
\hline 4491.405 & Fe II & 37 & -1.02 & 0.8 & 26 & -0.41 & 0.3 & 25 & -1.07 & 0.5 & 19 \\
\hline 4492.64 & Fe II & $6 \mathrm{~F}$ & 0.79 & 0.7 & 19 & 0.91 & 0.5 & 23 & 0.49 & 0.4 & 23 \\
\hline 4492.64 & $\mathrm{Fe}$ II & $6 \mathrm{~F}$ & 0.12 & 1.6 & 19 & 0.30 & 1.4 & 23 & 0.33 & 1.6 & 23 \\
\hline 4508.288 & Fe II & 38 & 1.67 & 0.9 & 24 & 1.52 & 0.8 & 21 & 1.84 & 0.7 & 15 \\
\hline 4508.288 & Fe II & 38 & 2.23 & 2.5 & 24 & 2.63 & 2.6 & 21 & 2.76 & 2.7 & 15 \\
\hline 4508.288 & Fe II & 38 & -0.79 & 0.8 & 29 & -0.67 & 0.3 & 24 & -1.58 & 0.5 & 19 \\
\hline 4509.61 & Fe II & $6 \mathrm{~F}$ & 0.42 & 0.7 & 19 & 0.45 & 0.5 & 19 & 0.20 & 0.4 & 19 \\
\hline 4509.61 & Fe II & $6 \mathrm{~F}$ & 0.16 & 1.6 & 19 & 0.31 & 1.4 & 19 & 0.21 & 1.6 & 19 \\
\hline 4514.9 & $\mathrm{Fe}$ II & $6 \mathrm{~F}$ & 0.82 & 0.7 & 21 & 0.87 & 0.4 & 22 & 0.76 & 0.4 & 20 \\
\hline 4514.9 & Fe II & $6 \mathrm{~F}$ & 0.16 & 1.6 & 21 & 0.28 & 1.4 & 22 & 0.98 & 1.6 & 20 \\
\hline 4515.339 & Fe II & 37 & 1.90 & 0.9 & 21 & 1.92 & 0.6 & 23 & 1.61 & 0.7 & 26 \\
\hline 4515.339 & Fe II & 37 & 2.27 & 2.7 & 21 & 2.50 & 2.6 & 23 & 2.24 & 2.7 & 26 \\
\hline 4515.339 & $\mathrm{Fe}$ II & 37 & -0.48 & 0.6 & 24 & -0.76 & 0.3 & 25 & -0.87 & 0.5 & 27 \\
\hline 4520.224 & Fe II & 37 & 2.56 & 0.9 & 20 & 1.61 & 0.7 & 21 & 1.72 & 0.7 & 14 \\
\hline 4520.224 & Fe II & 37 & 2.06 & 2.7 & 20 & 2.58 & 2.7 & 21 & 1.75 & 2.7 & 14 \\
\hline 4520.224 & Fe II & 37 & -1.05 & 0.8 & 24 & -0.30 & 0.4 & 24 & -0.61 & 0.5 & 18 \\
\hline 4522.634 & $\mathrm{Fe}$ II & 38 & 3.12 & 0.9 & 22 & 2.28 & 0.7 & 24 & 3.12 & 0.8 & 13 \\
\hline 4522.634 & Fe II & 38 & 2.41 & 2.7 & 22 & 3.41 & 2.7 & 24 & 2.24 & 2.7 & 13 \\
\hline 4522.634 & Fe II & 38 & -1.64 & 0.8 & 24 & -1.27 & 0.4 & 26 & -2.40 & 0.6 & 18 \\
\hline 4528.39 & Fe II & $6 \mathrm{~F}$ & 0.41 & 0.7 & 14 & 0.48 & 0.5 & 21 & 0.27 & 0.5 & 21 \\
\hline 4528.39 & $\mathrm{Fe}$ II & $6 \mathrm{~F}$ & 0.08 & 1.6 & 14 & 0.08 & 1.6 & 21 & 0.09 & 1.6 & 21 \\
\hline 4533.0 & Fe II & $6 \mathrm{~F}$ & 0.09 & 0.7 & 21 & 0.12 & 0.5 & 21 & 0.10 & 0.4 & 24 \\
\hline 4533.0 & Fe II & $6 \mathrm{~F}$ & 0.14 & 1.6 & 21 & 0.16 & 1.6 & 21 & 0.12 & 1.6 & 25 \\
\hline 4534.168 & Fe II & 37 & 0.45 & 0.9 & 23 & 0.43 & 0.7 & 23 & 0.34 & 0.7 & 23 \\
\hline 4534.168 & Fe II & 37 & 0.74 & 2.7 & 23 & 0.96 & 2.7 & 23 & 0.21 & 2.7 & 23 \\
\hline 4534.168 & Fe II & 37 & -0.09 & 0.6 & 25 & -0.25 & 0.5 & 25 & -0.19 & 0.5 & 23 \\
\hline 4541.524 & Fe II & 38 & 0.55 & 1.0 & 24 & 0.58 & 0.6 & 24 & 0.68 & 0.7 & 22 \\
\hline 4541.524 & Fe II & 38 & 0.53 & 2.7 & 24 & 0.84 & 2.5 & 24 & 0.53 & 2.7 & 22 \\
\hline 4541.524 & Fe II & 38 & -0.24 & 0.7 & 31 & -0.33 & 0.3 & 25 & -0.49 & 0.5 & 26 \\
\hline 4548.208 & Mn II & & 0.08 & 0.9 & 3 & 0.27 & 0.9 & 23 & 0.31 & 0.9 & 23 \\
\hline 4549.474 & $\mathrm{Fe}$ II & 38 & 4.91 & 0.9 & 20 & 3.11 & 0.8 & 19 & 4.80 & 1.0 & 19 \\
\hline 4549.474 & Fe II & 38 & 2.81 & 2.7 & 20 & 4.37 & 2.7 & 19 & 2.11 & 2.7 & 19 \\
\hline 4549.474 & Fe II & 38 & -2.64 & 0.7 & 23 & -1.19 & 0.4 & 22 & -4.05 & 0.7 & 27 \\
\hline 4549.617 & Ti II & 82 & 0.67 & 0.9 & 21 & 0.35 & 0.7 & 24 & 1.36 & 0.7 & 24 \\
\hline 4549.617 & Ti II & 82 & 0.80 & 2.7 & 21 & 0.55 & 2.7 & 24 & 0.51 & 2.7 & 24 \\
\hline 4554.988 & Cr II & 44 & 0.33 & 1.0 & 21 & 0.45 & 0.7 & 21 & 0.70 & 0.8 & 19 \\
\hline 4554.988 & Cr II & 44 & 0.51 & 2.7 & 21 & 0.68 & 2.7 & 21 & 0.40 & 2.7 & 19 \\
\hline 4554.988 & Cr II & 44 & -0.39 & 0.7 & 23 & -0.47 & 0.4 & 23 & -0.48 & 0.4 & 21 \\
\hline 4555.893 & $\mathrm{Fe}$ II & 37 & 3.32 & 1.0 & 20 & 3.12 & 0.7 & 24 & 3.04 & 0.8 & 22 \\
\hline 4555.893 & Fe II & 37 & 1.67 & 2.7 & 20 & 3.28 & 2.7 & 24 & 2.24 & 2.7 & 22 \\
\hline 4555.893 & $\mathrm{Fe}$ II & 37 & -0.62 & 0.7 & 24 & -1.04 & 0.4 & 25 & -1.14 & 0.5 & 25 \\
\hline 4558.65 & Cr II & 44 & 0.85 & 0.9 & 18 & 2.51 & 0.6 & 20 & 2.73 & 0.8 & 12 \\
\hline 4558.65 & Cr II & 44 & 0.95 & 2.5 & 18 & 3.73 & 3.5 & 20 & 1.05 & 2.7 & 12 \\
\hline 4558.65 & Cr II & 44 & -0.78 & 0.6 & 20 & -2.64 & 0.4 & 23 & -2.90 & 0.6 & 20 \\
\hline 4571.096 & Mg I & 1 & 1.55 & 1.3 & 32 & 1.74 & 1.0 & 29 & 1.16 & 1.0 & 27 \\
\hline 4571.096 & Mg I & 1 & -0.27 & 0.7 & 44 & -0.34 & 0.3 & 35 & -0.01 & 0.2 & 28 \\
\hline 4576.34 & Fe II & 38 & 1.06 & 1.0 & 20 & 0.95 & 0.7 & 22 & 1.02 & 1.0 & 23 \\
\hline 4576.34 & Fe II & 38 & 0.43 & 2.7 & 20 & 0.97 & 2.7 & 22 & 0.25 & 2.7 & 23 \\
\hline 4576.34 & $\mathrm{Fe}$ II & 38 & -0.10 & 0.7 & 24 & -0.24 & 0.4 & 24 & -0.08 & 0.4 & 28 \\
\hline 4581.395 & $\mathrm{Ca} \mathrm{I}$ & & -0.67 & 0.7 & 31 & -0.44 & 0.3 & -7 & -0.14 & 0.5 & -6 \\
\hline 4581.395 & $\mathrm{Ca} \mathrm{I}$ & & 1.78 & 1.5 & 26 & 1.04 & 0.6 & -5 & 0.66 & 0.9 & -5 \\
\hline 4582.835 & Fe II & 37 & 0.69 & 1.0 & 20 & 0.78 & 0.7 & 23 & 1.03 & 0.8 & 22 \\
\hline 4582.835 & Fe II & 37 & 0.13 & 2.7 & 20 & 0.41 & 2.7 & 23 & 1.33 & 2.7 & 22 \\
\hline 4582.835 & Fe II & 37 & -0.62 & 0.8 & 22 & -0.34 & 0.4 & 26 & -0.35 & 0.6 & 25 \\
\hline
\end{tabular}


G. Muratorio et al.: Analysis of the circumstellar environment of the B[e] star HD 45677 (FS Canis Majoris), Online Material p 6

Table 2. continued.

\begin{tabular}{|c|c|c|c|c|c|c|c|c|c|c|c|}
\hline$\overline{\overline{\text { IDENT }}}$ & & & $\overline{\overline{\text { F95 }}}$ & $\overline{\text { W95 }}$ & V95 & $\overline{\overline{\text { F99 }}}$ & $\overline{\text { W99 }}$ & V99 & $\overline{\text { F01 }}$ & $\overline{\overline{\text { W01 }}}$ & $\overline{\overline{\text { V01 }}}$ \\
\hline 4583.409 & Ti II & 39 & 0.74 & 1.0 & 21 & 0.57 & 0.7 & 20 & 0.96 & 0.8 & 22 \\
\hline 4583.837 & $\mathrm{Fe}$ II & 38 & 4.71 & 1.0 & 20 & 4.68 & 0.7 & 23 & 5.28 & 0.8 & 22 \\
\hline 4583.837 & $\mathrm{Fe}$ II & 38 & 5.83 & 2.7 & 20 & 6.83 & 2.7 & 23 & 5.26 & 2.7 & 22 \\
\hline 4583.837 & Fe II & 38 & -0.79 & 0.8 & 24 & -1.25 & 0.3 & 25 & -2.95 & 0.6 & 23 \\
\hline 4588.199 & Cr II & 44 & 0.86 & 1.1 & 19 & 1.51 & 0.6 & 24 & 1.71 & 0.8 & 18 \\
\hline 4588.199 & $\mathrm{Cr}$ II & 44 & 0.71 & 2.6 & 19 & 2.93 & 3.5 & 24 & 1.45 & 2.7 & 18 \\
\hline 4588.199 & Cr II & 44 & -0.52 & 0.6 & 21 & -1.66 & 0.4 & 26 & -1.93 & 0.5 & 22 \\
\hline 4616.629 & $\mathrm{Cr}$ II & 44 & 0.29 & 1.0 & 25 & 0.43 & 0.7 & 23 & & & \\
\hline 4616.629 & Cr II & 44 & 0.26 & 2.7 & 25 & 0.82 & 2.7 & 23 & & & \\
\hline 4616.629 & Cr II & 44 & -0.27 & 0.7 & 29 & -0.32 & 0.4 & 26 & & & \\
\hline 4618.803 & Cr II & 44 & 0.47 & 1.0 & 17 & 1.39 & 0.6 & 28 & & & \\
\hline 4618.803 & Cr II & 44 & 0.83 & 2.7 & 17 & 1.83 & 2.7 & 28 & & & \\
\hline 4618.803 & Cr II & 44 & -0.47 & 0.7 & 22 & -1.59 & 0.5 & 31 & & & \\
\hline 4620.521 & $\mathrm{Fe}$ II & 38 & 0.38 & 1.0 & 18 & 0.46 & 0.6 & 27 & & & \\
\hline 4620.521 & $\mathrm{Fe}$ II & 38 & 0.52 & 2.7 & 18 & 0.37 & 2.7 & 27 & & & \\
\hline 4620.521 & $\mathrm{Fe}$ II & 38 & -0.29 & 0.7 & 27 & -0.20 & 0.4 & 32 & & & \\
\hline 4628.77 & Ni II & $3 \mathrm{~F}$ & 0.38 & 0.7 & -27 & 0.47 & 0.4 & -16 & & & \\
\hline 4629.279 & Ti II & 38 & 1.12 & 1.0 & 21 & 0.88 & 0.7 & 29 & & & \\
\hline 4629.339 & $\mathrm{Fe}$ II & 37 & 3.55 & 1.0 & 20 & 3.38 & 0.7 & 32 & & & \\
\hline 4629.339 & $\mathrm{Fe}$ II & 37 & 1.53 & 2.7 & 20 & 3.13 & 2.7 & 32 & & & \\
\hline 4629.339 & $\mathrm{Fe}$ II & 37 & -0.55 & 0.9 & 24 & -0.91 & 0.3 & 34 & & & \\
\hline 4634.07 & Cr II & 44 & 0.74 & 1.0 & 27 & 0.81 & 1.0 & 27 & & & \\
\hline 4634.07 & Cr II & 44 & 0.34 & 2.7 & 23 & 0.82 & 2.7 & 27 & & & \\
\hline 4634.07 & Cr II & 44 & -0.69 & 0.8 & 25 & -0.55 & 0.4 & 34 & & & \\
\hline 4639.68 & $\mathrm{Fe}$ II & $4 \mathrm{~F}$ & 1.38 & 0.7 & 16 & 1.37 & 0.4 & 30 & & & \\
\hline 4639.68 & $\mathrm{Fe}$ II & $4 \mathrm{~F}$ & 0.40 & 1.6 & 16 & 0.62 & 1.6 & 30 & & & \\
\hline 4664.45 & $\mathrm{Fe}$ II & $4 \mathrm{~F}$ & 0.40 & 0.7 & 16 & 0.54 & 0.7 & 16 & & & \\
\hline 4664.45 & $\mathrm{Fe}$ II & $4 \mathrm{~F}$ & 0.31 & 1.6 & 16 & 0.24 & 1.6 & 16 & & & \\
\hline 4666.758 & $\mathrm{Fe}$ II & 37 & 0.69 & 1.0 & 18 & 0.99 & 1.0 & 17 & & & \\
\hline 4666.758 & $\mathrm{Fe}$ II & 37 & 0.27 & 2.7 & 18 & 0.67 & 2.7 & 17 & & & \\
\hline 4666.758 & $\mathrm{Fe}$ II & 37 & -0.11 & 0.8 & 20 & -0.18 & 0.8 & 20 & & & \\
\hline 4713.139 & He I & 12 & -2.19 & 1.9 & 30 & -2.14 & 1.6 & 41 & & & \\
\hline 4728.07 & $\mathrm{Fe}$ II & $4 \mathrm{~F}$ & 2.82 & 0.7 & 21 & 2.65 & 0.8 & 22 & & & \\
\hline 4728.07 & $\mathrm{Fe}$ II & $4 \mathrm{~F}$ & 0.32 & 1.6 & 22 & 1.19 & 1.6 & 22 & & & \\
\hline 4731.453 & $\mathrm{Fe}$ II & 43 & 0.22 & 0.9 & 21 & & & & & & \\
\hline 4731.453 & $\mathrm{Fe}$ II & 43 & 0.28 & 1.8 & 24 & & & & & & \\
\hline 4772.07 & $\mathrm{Fe}$ II & $4 \mathrm{~F}$ & 0.16 & 0.7 & 20 & & & & & & \\
\hline 4774.74 & $\mathrm{Fe}$ II & $20 \mathrm{~F}$ & 1.10 & 0.7 & 19 & 1.55 & 0.7 & 18 & & & \\
\hline 4774.74 & $\mathrm{Fe}$ II & $20 \mathrm{~F}$ & 0.36 & 1.6 & 19 & 0.56 & 1.6 & 18 & & & \\
\hline 4798.28 & $\mathrm{Fe}$ II & $4 \mathrm{~F}$ & 0.36 & 0.7 & 18 & 0.71 & 0.8 & 29 & & & \\
\hline 4798.28 & $\mathrm{Fe}$ II & $4 \mathrm{~F}$ & 0.16 & 1.6 & 18 & 0.28 & 1.6 & 29 & & & \\
\hline 4814.55 & $\mathrm{Fe}$ II & $20 \mathrm{~F}$ & 3.54 & 0.7 & 20 & 4.04 & 0.8 & 19 & & & \\
\hline 4814.55 & $\mathrm{Fe}$ II & $20 \mathrm{~F}$ & 1.32 & 1.6 & 20 & 1.98 & 1.6 & 20 & & & \\
\hline 4824.127 & Cr II & 30 & 0.76 & 1.0 & 24 & 1.56 & 0.9 & 19 & & & \\
\hline 4824.127 & Cr II & 30 & 0.97 & 2.8 & 24 & 2.48 & 2.5 & 19 & & & \\
\hline 4824.127 & Cr II & 30 & -0.45 & 0.6 & 29 & -1.53 & 0.8 & 23 & & & \\
\hline 4848.235 & Cr II & 30 & 0.69 & 1.0 & 30 & 1.04 & 1.0 & 14 & & & \\
\hline 4848.235 & Cr II & 30 & 0.42 & 2.8 & 30 & 1.01 & 2.5 & 14 & & & \\
\hline 4848.235 & Cr II & 30 & -0.40 & 0.7 & 31 & -0.85 & 0.6 & 18 & & & \\
\hline 4861.332 & $\mathrm{H} \beta$ & & -49.5 & 1.6 & -51 & -50.1 & 1.8 & -45 & & & \\
\hline 4861.332 & $\mathrm{H} \beta$ & & -16.0 & 17.0 & 23 & -8.4 & 17. & 24 & & & \\
\hline 4861.332 & $\mathrm{H} \beta$ & & 237. & 3.1 & 23 & 224 & 3.2 & 24 & & & \\
\hline 4861.332 & $\mathrm{H} \beta$ & & -78 & 1.3 & 25 & -75 & 1.4 & 28 & & & \\
\hline
\end{tabular}


G. Muratorio et al.: Analysis of the circumstellar environment of the B[e] star HD 45677 (FS Canis Majoris), Online Material $p 7$

Table 2. continued.

\begin{tabular}{|c|c|c|c|c|c|c|c|c|c|c|c|}
\hline$\overline{\text { IDENT }}$ & & & $\overline{\text { F95 }}$ & $\overline{\text { W95 }}$ & $\overline{\text { V95 }}$ & $\overline{\overline{\text { F99 }}}$ & $\overline{\overline{\text { W99 }}}$ & $\overline{\overline{\text { V99 }}}$ & $\overline{\overline{\text { F01 }}}$ & $\overline{\overline{\text { W01 }}}$ & $\overline{\text { V01 }}$ \\
\hline 4874.49 & Fe II & $20 \mathrm{~F}$ & 1.23 & 0.7 & 20 & 1.68 & 0.7 & 23 & & & \\
\hline 4874.49 & $\mathrm{Fe}$ II & $20 \mathrm{~F}$ & 0.66 & 1.6 & 20 & 0.24 & 1.6 & 23 & & & \\
\hline 4889.63 & $\mathrm{Fe}$ II & $4 \mathrm{~F}$ & 3.30 & 0.7 & 17 & 3.46 & 0.8 & 21 & & & \\
\hline 4889.63 & Fe II & $4 \mathrm{~F}$ & 0.48 & 1.6 & 18 & 0.71 & 1.6 & 21 & & & \\
\hline 4898.607 & $\mathrm{Fe}$ II & $97 \mathrm{~F}$ & 0.95 & 0.7 & 16 & 1.25 & 0.8 & 26 & & & \\
\hline 4905.35 & Fe II & $20 \mathrm{~F}$ & 1.76 & 0.7 & 17 & 2.67 & 0.8 & 23 & & & \\
\hline 4905.35 & $\mathrm{Fe}$ II & $20 \mathrm{~F}$ & 0.79 & 1.6 & 17 & 0.88 & 1.6 & 24 & & & \\
\hline 4921.931 & $\mathrm{He} \mathrm{I}$ & 48 & -6.39 & 3.9 & 32 & -2.07 & 2.5 & 23 & & & \\
\hline 4923.927 & Fe II & 42 & 10.45 & 1.0 & 25 & 5.55 & 1.0 & 30 & & & \\
\hline 4923.927 & $\mathrm{Fe}$ II & 42 & 8.30 & 2.9 & 25 & 10.01 & 2.5 & 30 & & & \\
\hline 4923.927 & $\mathrm{Fe}$ II & 42 & -3.66 & 0.8 & 28 & -2.25 & 0.7 & 37 & & & \\
\hline 4947.38 & $\mathrm{Fe}$ II & $20 \mathrm{~F}$ & 0.83 & 1.2 & 21 & 0.74 & 0.7 & 23 & & & \\
\hline 4950.74 & $\mathrm{Fe}$ II & $20 \mathrm{~F}$ & 1.33 & 0.8 & 20 & 1.21 & 0.7 & 24 & & & \\
\hline 4973.39 & $\mathrm{Fe}$ II & $20 \mathrm{~F}$ & 1.41 & 0.8 & 20 & 1.25 & 0.8 & 19 & & & \\
\hline 5005.52 & $\mathrm{Fe}$ II & $20 \mathrm{~F}$ & 0.74 & 0.8 & 20 & 1.19 & 0.8 & 22 & & & \\
\hline 5005.52 & $\mathrm{Fe}$ II & $20 \mathrm{~F}$ & & & & 0.32 & 1.6 & 22 & & & \\
\hline 5006.65 & $\mathrm{Fe}$ II & $4 \mathrm{~F}$ & 0.22 & 0.8 & 14 & 0.48 & 0.8 & 29 & & & \\
\hline 5006.65 & $\mathrm{Fe}$ II & $4 \mathrm{~F}$ & & & & 0.24 & 1.6 & 29 & & & \\
\hline 5015.678 & $\mathrm{He} \mathrm{I}$ & 4 & -4.52 & 3.4 & 29 & -1.99 & 2.8 & 28 & & & \\
\hline 5018.44 & $\mathrm{Fe}$ II & 42 & 9.99 & 1.0 & 25 & 8.32 & 1.1 & 31 & & & \\
\hline 5018.44 & $\mathrm{Fe}$ II & 42 & 11.83 & 2.5 & 25 & 15.09 & 2.9 & 31 & & & \\
\hline 5018.44 & $\mathrm{Fe}$ II & 42 & -3.79 & 0.8 & 25 & -3.20 & 0.7 & 37 & & & \\
\hline 5020.24 & $\mathrm{Fe}$ II & $20 \mathrm{~F}$ & 1.20 & 0.7 & 20 & 1.06 & 0.8 & 22 & & & \\
\hline 5043.53 & Fe II & $20 \mathrm{~F}$ & 0.79 & 1.0 & 17 & 0.66 & 0.7 & 16 & & & \\
\hline 5047.738 & $\mathrm{He} \mathrm{I}$ & 47 & -0.75 & 1.4 & -7 & -1.19 & 1.6 & 28 & & & \\
\hline 5107.95 & $\mathrm{Fe}$ II & $18 \mathrm{~F}$ & 0.99 & 0.9 & 20 & & & & & & \\
\hline 5111.63 & $\mathrm{Fe}$ II & $19 \mathrm{~F}$ & 1.50 & 0.9 & 19 & & & & & & \\
\hline 5158.0 & $\mathrm{Fe}$ II & $18 \mathrm{~F}$ & 1.50 & 0.8 & 21 & 1.39 & 0.4 & 23 & 1.15 & 0.5 & 23 \\
\hline 5158.0 & $\mathrm{Fe}$ II & $18 \mathrm{~F}$ & 0.51 & 1.7 & 21 & 0.82 & 1.6 & 23 & 0.63 & 1.6 & 18 \\
\hline 5158.81 & $\mathrm{Fe}$ II & $19 \mathrm{~F}$ & 4.63 & 0.8 & 22 & 4.74 & 0.5 & 21 & 3.36 & 0.5 & 20 \\
\hline 5158.81 & $\mathrm{Fe}$ II & $19 \mathrm{~F}$ & 1.35 & 1.7 & 22 & 1.64 & 1.6 & 21 & 0.95 & 1.6 & 28 \\
\hline 5163.951 & $\mathrm{Fe}$ II & $35 \mathrm{~F}$ & 1.48 & 0.8 & 24 & 1.38 & 0.4 & 22 & 0.80 & 0.5 & 23 \\
\hline 5163.951 & Fe II & $35 \mathrm{~F}$ & 0.34 & 1.7 & 24 & 0.46 & 1.5 & 22 & 0.39 & 1.6 & 23 \\
\hline 5167.321 & Mg I & 2 & 0.45 & 0.9 & 27 & 0.36 & 0.7 & 27 & 0.41 & 0.7 & 28 \\
\hline 5167.321 & $\mathrm{Mg} \mathrm{I}$ & 2 & 1.55 & 3.1 & 27 & 1.59 & 3.1 & 27 & 1.22 & 3.1 & 28 \\
\hline 5169.033 & $\mathrm{Fe}$ II & 42 & 7.60 & 0.9 & 27 & 7.17 & 0.7 & 26 & 7.99 & 0.9 & 13 \\
\hline 5169.033 & $\mathrm{Fe}$ II & 42 & 10.77 & 3.1 & 27 & 12.70 & 3.1 & 26 & 8.25 & 3.1 & 13 \\
\hline 5169.033 & $\mathrm{Fe}$ II & 42 & -3.13 & 0.7 & 28 & -4.18 & 0.5 & 28 & -8.51 & 0.8 & 15 \\
\hline 5172.684 & Mg I & 2 & 0.40 & 0.9 & 27 & 0.43 & 0.7 & 26 & 0.14 & 0.7 & 25 \\
\hline 5172.684 & $\mathrm{Mg} \mathrm{I}$ & 2 & 0.92 & 3.1 & 27 & 1.90 & 3.1 & 26 & 1.22 & 3.1 & 25 \\
\hline 5181.97 & $\mathrm{Fe}$ II & $18 \mathrm{~F}$ & 0.88 & 0.8 & 22 & 1.32 & 0.6 & 21 & 0.77 & 0.6 & 22 \\
\hline 5181.97 & $\mathrm{Fe}$ II & $18 \mathrm{~F}$ & 0.42 & 1.7 & 22 & 0.39 & 1.6 & 21 & 0.31 & 1.6 & 22 \\
\hline 5183.604 & Mg I & 2 & 0.78 & 1.1 & 25 & 1.69 & 1.1 & 21 & 1.63 & 1.1 & 17 \\
\hline 5183.604 & $\mathrm{Mg} \mathrm{I}$ & 2 & 0.31 & 3.1 & 25 & 0.63 & 3.1 & 21 & 0.61 & 3.1 & 17 \\
\hline 5183.604 & $\mathrm{Mg} \mathrm{I}$ & 2 & -0.27 & 0.8 & 28 & -0.85 & 0.6 & 25 & -1.14 & 0.7 & 22 \\
\hline 5184.788 & $\mathrm{Fe}$ II & $19 \mathrm{~F}$ & 0.38 & 0.8 & 17 & 0.46 & 0.6 & 23 & 0.22 & 0.6 & 21 \\
\hline 5184.788 & $\mathrm{Fe}$ II & $19 \mathrm{~F}$ & 0.10 & 1.7 & 17 & 0.12 & 1.6 & 23 & 0.08 & 1.6 & 21 \\
\hline 5197.577 & $\mathrm{Fe}$ II & 49 & 2.19 & 0.9 & 23 & 2.86 & 0.8 & 23 & 2.32 & 0.9 & 20 \\
\hline 5197.577 & $\mathrm{Fe}$ II & 49 & 3.58 & 3.1 & 23 & 2.00 & 3.1 & 23 & 2.35 & 3.1 & 20 \\
\hline 5197.577 & $\mathrm{Fe}$ II & 49 & -0.40 & 0.7 & 26 & -0.61 & 0.4 & 24 & -0.67 & 0.4 & 22 \\
\hline 5199.173 & $\mathrm{Fe}$ II & $35 \mathrm{~F}$ & 0.36 & 0.7 & 22 & 0.57 & 0.5 & 21 & 0.44 & 0.6 & 23 \\
\hline 5199.173 & Fe II & $35 \mathrm{~F}$ & 0.32 & 1.6 & 22 & 0.16 & 1.6 & 21 & 0.25 & 1.7 & 23 \\
\hline 5214.489 & $\mathrm{Fe}$ II & & & & & & & & 0.14 & 1.1 & 13 \\
\hline 5214.489 & Fe II & & & & & & & & 0.45 & 3.1 & 13 \\
\hline 5216.863 & $\mathrm{Fe}$ II & & & & & & & & 0.14 & 1.1 & 23 \\
\hline 5216.863 & $\mathrm{Fe}$ II & & & & & & & & 0.60 & 3.1 & 23 \\
\hline
\end{tabular}


G. Muratorio et al.: Analysis of the circumstellar environment of the B[e] star HD 45677 (FS Canis Majoris), Online Material p 8

Table 2. continued.

\begin{tabular}{|c|c|c|c|c|c|c|c|c|c|c|c|}
\hline$\overline{\overline{\text { IDENT }}}$ & & & $\overline{\overline{\text { F95 }}}$ & $\overline{\text { W95 }}$ & V95 & $\overline{\text { F99 }}$ & $\overline{\text { W99 }}$ & V99 & $\overline{\overline{\text { F01 }}}$ & $\overline{\text { W01 }}$ & $\overline{\overline{\text { V01 }}}$ \\
\hline 5220.06 & Fe II & $19 \mathrm{~F}$ & 1.18 & 0.8 & 20 & 1.18 & 0.5 & 22 & 0.82 & 0.6 & 22 \\
\hline 5220.06 & $\mathrm{Fe}$ II & $19 \mathrm{~F}$ & 0.34 & 1.7 & 20 & 0.52 & 1.7 & 22 & 0.47 & 1.6 & 22 \\
\hline 5234.625 & $\mathrm{Fe}$ II & 49 & 2.69 & 0.9 & 27 & 3.12 & 0.9 & 23 & 2.36 & 0.9 & 18 \\
\hline 5234.625 & Fe II & 49 & 2.77 & 3.1 & 27 & 2.77 & 3.0 & 23 & 2.60 & 3.1 & 18 \\
\hline 5234.625 & $\mathrm{Fe}$ II & 49 & -0.64 & 0.8 & 27 & -0.64 & 0.4 & 25 & -0.87 & 0.6 & 22 \\
\hline 5237.329 & $\mathrm{Cr}$ II & 43 & 0.38 & 0.9 & 24 & 0.83 & 0.9 & 23 & 0.56 & 0.9 & 24 \\
\hline 5237.329 & Cr II & 43 & 0.77 & 3.1 & 24 & 0.62 & 3.0 & 23 & 0.71 & 3.1 & 24 \\
\hline 5237.329 & Cr II & 43 & -0.26 & 0.8 & 26 & -0.40 & 0.4 & 25 & -0.37 & 0.5 & 24 \\
\hline 5247.952 & Fe II & & & & & & & & 0.69 & 3.1 & 22 \\
\hline 5247.952 & $\mathrm{Fe}$ II & & & & & & & & 0.14 & 1.1 & 22 \\
\hline 5251.233 & Fe II & & & & & & & & 0.39 & 3.1 & 25 \\
\hline 5251.233 & $\mathrm{Fe}$ II & & & & & & & & 0.11 & 1.1 & 25 \\
\hline 5254.929 & $\mathrm{Fe}$ II & 49 & 0.48 & 1.1 & 30 & 0.58 & 1.1 & 26 & 0.54 & 1.1 & 24 \\
\hline 5254.929 & $\mathrm{Fe}$ II & 49 & 0.30 & 3.1 & 30 & 0.41 & 3.1 & 26 & 0.24 & 3.1 & 24 \\
\hline 5254.929 & $\mathrm{Fe}$ II & 49 & -0.13 & 0.9 & 35 & -0.14 & 0.6 & 29 & -0.14 & 0.5 & 26 \\
\hline 5261.61 & $\mathrm{Fe}$ II & $19 \mathrm{~F}$ & 3.35 & 0.7 & 25 & 3.65 & 0.5 & 23 & 2.45 & 0.5 & 23 \\
\hline 5261.61 & $\mathrm{Fe}$ II & $19 \mathrm{~F}$ & 0.84 & 1.7 & 25 & 1.54 & 1.5 & 23 & 1.08 & 1.5 & 28 \\
\hline 5264.177 & $\mathrm{Fe}$ II & & & & & & & & 0.15 & 3.1 & 24 \\
\hline 5268.88 & $\mathrm{Fe}$ II & $18 \mathrm{~F}$ & 0.92 & 0.7 & 25 & 1.09 & 0.5 & 22 & 1.14 & 0.6 & 19 \\
\hline 5268.88 & Fe II & $18 \mathrm{~F}$ & 0.17 & 1.7 & 25 & 0.31 & 1.5 & 35 & 0.16 & 1.6 & 19 \\
\hline 5273.38 & $\mathrm{Fe}$ II & $18 \mathrm{~F}$ & 3.05 & 0.8 & 23 & 3.61 & 0.6 & 22 & 2.14 & 0.5 & 20 \\
\hline 5273.38 & $\mathrm{Fe}$ II & $18 \mathrm{~F}$ & 0.81 & 1.7 & 23 & 1.15 & 1.6 & 22 & 0.69 & 1.6 & 20 \\
\hline 5276.002 & $\mathrm{Fe}$ II & 49 & 4.79 & 1.0 & 29 & 5.06 & 0.9 & 24 & 3.94 & 0.9 & 19 \\
\hline 5276.002 & $\mathrm{Fe}$ II & 49 & 2.46 & 3.1 & 29 & 2.86 & 3.1 & 24 & 1.99 & 3.1 & 19 \\
\hline 5276.002 & $\mathrm{Fe}$ II & 49 & -1.60 & 0.8 & 35 & -1.52 & 0.6 & 27 & -1.72 & 0.7 & 22 \\
\hline 5278.374 & $\mathrm{Fe}$ II & $35 \mathrm{~F}$ & 0.11 & 0.8 & 3 & 0.26 & 0.6 & 26 & 0.14 & 0.6 & 19 \\
\hline 5278.374 & Fe II & $35 \mathrm{~F}$ & 0.08 & 1.7 & 3 & 0.16 & 1.6 & 26 & 0.11 & 1.6 & 19 \\
\hline 5283.109 & $\mathrm{Fe}$ II & $35 \mathrm{~F}$ & 0.23 & 0.7 & 28 & 0.34 & 0.5 & 22 & 0.25 & 0.5 & 23 \\
\hline 5283.109 & Fe II & $35 \mathrm{~F}$ & 0.07 & 1.7 & 28 & 0.09 & 1.6 & 21 & 0.09 & 1.6 & 23 \\
\hline 5284.109 & $\mathrm{Fe}$ II & 41 & 0.65 & 1.1 & 30 & 0.57 & 0.9 & 21 & 0.59 & 0.9 & 23 \\
\hline 5284.109 & $\mathrm{Fe}$ II & 41 & 0.31 & 3.1 & 30 & 0.32 & 3.1 & 21 & 0.28 & 3.1 & 23 \\
\hline 5284.109 & $\mathrm{Fe}$ II & 41 & -0.18 & 0.8 & 31 & -0.18 & 0.6 & 23 & -0.30 & 0.6 & 23 \\
\hline 5296.84 & $\mathrm{Fe}$ II & $19 \mathrm{~F}$ & 0.64 & 0.6 & 25 & 1.03 & 0.5 & 22 & 0.69 & 0.5 & 22 \\
\hline 5296.84 & $\mathrm{Fe}$ II & $19 \mathrm{~F}$ & 0.25 & 1.7 & 25 & 0.25 & 1.6 & 22 & 0.21 & 1.6 & 22 \\
\hline 5316.615 & $\mathrm{Fe}$ II & 49 & 7.30 & 1.1 & 39 & 8.67 & 0.9 & 24 & 6.11 & 1.0 & 18 \\
\hline 5316.615 & Fe II & 49 & 3.83 & 3.1 & 39 & 4.45 & 3.1 & 24 & 3.36 & 3.1 & 18 \\
\hline 5316.615 & $\mathrm{Fe}$ II & 49 & -0.67 & 0.9 & 40 & -1.83 & 0.5 & 26 & -0.66 & 0.5 & 19 \\
\hline 5316.784 & $\mathrm{Fe}$ II & 48 & 1.90 & 1.1 & 32 & 1.74 & 0.9 & 22 & 1.33 & 0.9 & 22 \\
\hline 5316.784 & $\mathrm{Fe}$ II & 48 & 1.30 & 3.1 & 32 & 1.59 & 3.1 & 22 & 1.53 & 3.1 & 22 \\
\hline 5316.784 & $\mathrm{Fe}$ II & 48 & -0.31 & 0.9 & 35 & -0.59 & 0.5 & 24 & -0.58 & 0.5 & 23 \\
\hline 5325.553 & $\mathrm{Fe}$ II & 49 & & & & 0.87 & 0.9 & 26 & 0.98 & 0.9 & 22 \\
\hline 5325.553 & $\mathrm{Fe}$ II & 49 & & & & 0.38 & 3.1 & 26 & 0.31 & 3.1 & 22 \\
\hline 5325.553 & $\mathrm{Fe}$ II & 49 & & & & -0.44 & 0.5 & 30 & -0.59 & 0.6 & 25 \\
\hline 5333.65 & $\mathrm{Fe}$ II & $19 \mathrm{~F}$ & 2.54 & 0.7 & 24 & 2.92 & 0.5 & 23 & 1.88 & 0.5 & 21 \\
\hline 5333.65 & Fe II & $19 \mathrm{~F}$ & 0.76 & 1.7 & 24 & 0.82 & 1.6 & 23 & 0.67 & 1.6 & 21 \\
\hline 5347.67 & $\mathrm{Fe}$ II & $18 \mathrm{~F}$ & 0.34 & 0.8 & 17 & 0.39 & 0.6 & 18 & 0.31 & 0.6 & 19 \\
\hline 5347.67 & $\mathrm{Fe}$ II & $18 \mathrm{~F}$ & 0.34 & 1.7 & 17 & 0.25 & 1.6 & 21 & & & \\
\hline 5362.869 & $\mathrm{Fe}$ II & 48 & 1.23 & 1.1 & 25 & 1.19 & 1.1 & 27 & & & \\
\hline 5362.869 & Fe II & 48 & 0.77 & 3.1 & 25 & 0.88 & 3.2 & 27 & & & \\
\hline 5362.869 & Fe II & 48 & -0.24 & 0.8 & 28 & -0.24 & 0.8 & 30 & & & \\
\hline 5366.208 & $\mathrm{Fe}$ II & 97 & 0.16 & 1.1 & 36 & 0.50 & 1.5 & 26 & & & \\
\hline 5376.47 & $\mathrm{Fe}$ II & $19 \mathrm{~F}$ & 2.43 & 0.7 & 20 & 2.36 & 0.7 & 22 & & & \\
\hline 5376.47 & $\mathrm{Fe}$ II & $19 \mathrm{~F}$ & 0.59 & 1.7 & 21 & 0.58 & 1.6 & 22 & & & \\
\hline 5412.641 & Fe II & $17 \mathrm{~F}$ & 0.86 & 0.7 & 22 & 0.88 & 0.7 & 24 & & & \\
\hline 5412.641 & $\mathrm{Fe}$ II & $17 \mathrm{~F}$ & 0.32 & 1.7 & 22 & 0.35 & 1.6 & 24 & & & \\
\hline
\end{tabular}


G. Muratorio et al.: Analysis of the circumstellar environment of the B[e] star HD 45677 (FS Canis Majoris), Online Material p 9

Table 2. continued.

\begin{tabular}{|c|c|c|c|c|c|c|c|c|c|c|c|}
\hline$\overline{\overline{\text { IDENT }}}$ & & & $\overline{\text { F95 }}$ & $\overline{\text { W95 }}$ & V95 & F99 & W99 & "V99 & $\overline{\text { F01 }}$ & W01 & $\overline{\mathrm{V} \text { V01 }}$ \\
\hline 5414.862 & Cr II & & 0.44 & 0.8 & 26 & & & & & & \\
\hline 5425.257 & $\mathrm{Fe}$ II & 49 & 0.59 & 1.1 & 27 & 0.60 & 1.1 & 23 & & & \\
\hline 5425.257 & $\mathrm{Fe} I I$ & 49 & 0.08 & 3.2 & 27 & 0.24 & 3.2 & 23 & & & \\
\hline 5425.257 & $\mathrm{Fe}$ II & 49 & -0.12 & 0.8 & 33 & -0.16 & 0.8 & 23 & & & \\
\hline 5433.15 & $\mathrm{Fe} I I$ & $18 \mathrm{~F}$ & 0.93 & 0.7 & 19 & 0.82 & 0.6 & 22 & & & \\
\hline 5433.15 & $\mathrm{Fe} I I$ & $18 \mathrm{~F}$ & 0.21 & 1.7 & 19 & 0.24 & 1.6 & 24 & & & \\
\hline 5453.855 & S II & 6 & -0.81 & 2.2 & 27 & -0.52 & 1.3 & 22 & & & \\
\hline 5456.442 & Si II & & -0.14 & 2.2 & 26 & -0.26 & 1.3 & 25 & & & \\
\hline 5473.596 & V I & & 0.19 & 0.9 & 8 & & & & & & \\
\hline 5477.25 & $\mathrm{Fe} I I$ & $34 \mathrm{~F}$ & 0.56 & 0.8 & 20 & 0.52 & 0.7 & 21 & & & \\
\hline 5477.25 & $\mathrm{Fe}$ II & $34 \mathrm{~F}$ & 0.21 & 1.7 & 20 & 0.29 & 1.6 & 20 & & & \\
\hline 5492.399 & $\mathrm{Fe} I I$ & & 0.22 & 1.1 & 26 & & & & & & \\
\hline 5492.399 & $\mathrm{Fe}$ II & & 0.11 & 3.2 & 26 & & & & & & \\
\hline 5494.569 & $\mathrm{Ca} \mathrm{I}$ & & 0.22 & 0.9 & 29 & 0.22 & 0.9 & 29 & & & \\
\hline 5495.82 & $\mathrm{Fe}$ II & $17 \mathrm{~F}$ & 0.40 & 0.7 & 25 & 0.43 & 0.7 & 26 & & & \\
\hline 5495.82 & $\mathrm{Fe} I I$ & $17 \mathrm{~F}$ & 0.27 & 1.8 & 25 & 0.10 & 1.8 & 26 & & & \\
\hline 5527.331 & $\mathrm{Fe} I I$ & $17 \mathrm{~F}$ & 1.77 & 0.8 & 24 & 1.68 & 0.8 & 25 & & & \\
\hline 5527.331 & $\mathrm{Fe} I I$ & $17 \mathrm{~F}$ & 0.54 & 1.8 & 24 & 0.72 & 1.6 & 25 & & & \\
\hline 5534.847 & $\mathrm{Fe}$ II & 55 & 1.30 & 1.1 & 24 & 1.23 & 1.1 & 22 & & & \\
\hline 5534.847 & $\mathrm{Fe}$ II & 55 & 1.02 & 3.2 & 25 & 0.80 & 3.2 & 22 & & & \\
\hline 5534.847 & $\mathrm{Fe} I I$ & 55 & -0.21 & 0.8 & 28 & -0.12 & 0.8 & 25 & & & \\
\hline 5551.31 & $\mathrm{Fe}$ II & $39 \mathrm{~F}$ & 0.50 & 0.9 & 23 & 0.38 & 0.9 & 22 & & & \\
\hline 5556.31 & $\mathrm{Fe}$ II & $18 \mathrm{~F}$ & 0.22 & 0.8 & 21 & 0.19 & 0.8 & 27 & & & \\
\hline 5577.35 & O I & F & 1.21 & 1.2 & 15 & 1.43 & 1.5 & 14 & & & \\
\hline 5580.82 & $\mathrm{Fe} I I$ & $39 \mathrm{~F}$ & 0.41 & 0.9 & 23 & 0.33 & 0.8 & 12 & & & \\
\hline 5587.114 & $\mathrm{Fe} I I$ & 224 & 0.21 & 2.0 & 24 & & & & & & \\
\hline 5588.15 & $\mathrm{Fe}$ II & $39 \mathrm{~F}$ & 0.17 & 0.8 & 23 & 0.18 & 0.8 & 9 & & & \\
\hline 5613.268 & $\mathrm{Fe} I I$ & $39 \mathrm{~F}$ & 0.30 & 1.0 & 24 & 0.21 & 0.8 & 29 & & & \\
\hline 5627.249 & $\mathrm{Fe}$ II & $98 \mathrm{~F}$ & 0.27 & 1.2 & 23 & 0.24 & 0.8 & 28 & & & \\
\hline 5639.477 & Si II & 9 & -0.34 & 1.7 & 24 & -0.26 & 1.3 & 24 & & & \\
\hline 5639.977 & S II & 14 & -0.17 & 1.7 & 22 & -0.13 & 1.3 & 24 & & & \\
\hline 5640.346 & S II & 11 & -0.17 & 1.7 & 24 & -0.13 & 1.3 & 24 & & & \\
\hline 5640.554 & C II & & -0.42 & 1.7 & 25 & -0.32 & 1.30 & 24 & & & \\
\hline 5645.681 & S II & 6 & -0.22 & 2.2 & 25 & -0.30 & 2.0 & 25 & & & \\
\hline 5648.072 & C II & & -0.11 & 2.2 & 26 & -0.40 & 2.0 & 26 & & & \\
\hline 5654.850 & $\mathrm{Fe}$ II & $17 \mathrm{~F}$ & 0.20 & 0.8 & 20 & 0.27 & 0.8 & 31 & & & \\
\hline 5673.211 & $\mathrm{Fe} I I$ & $97 \mathrm{~F}$ & 0.75 & 1.0 & 19 & 0.65 & 0.8 & 15 & & & \\
\hline 5718.215 & $\mathrm{Fe} I I$ & $39 \mathrm{~F}$ & 0.15 & 0.8 & 18 & 0.11 & 0.8 & 36 & & & \\
\hline 5718.215 & $\mathrm{Fe} I I$ & $39 \mathrm{~F}$ & 0.05 & 1.8 & 18 & 0.05 & 1.8 & 36 & & & \\
\hline 5745.7 & $\mathrm{Fe}$ II & $17 \mathrm{~F}$ & 0.12 & 0.8 & 25 & 0.06 & 0.8 & 26 & & & \\
\hline 5745.7 & $\mathrm{Fe}$ II & $17 \mathrm{~F}$ & 0.04 & 1.8 & 25 & 0.04 & 1.6 & 26 & & & \\
\hline 5746.96 & $\mathrm{Fe}$ II & $34 \mathrm{~F}$ & 1.09 & 0.7 & 21 & 1.25 & 0.8 & 22 & & & \\
\hline 5746.96 & Fe II & $34 \mathrm{~F}$ & 0.35 & 2.0 & 21 & 0.04 & 1.6 & 21 & & & \\
\hline 5754.8 & Ni II & $3 F$ & 1.26 & 1.0 & 11 & 1.37 & 1.0 & 12 & & & \\
\hline 5835.449 & $\mathrm{Fe}$ II & $44 \mathrm{~F}$ & 0.30 & 1.2 & 28 & 0.49 & 0.9 & 22 & 0.35 & 0.5 & 23 \\
\hline 5835.449 & $\mathrm{Fe} I I$ & $44 \mathrm{~F}$ & 0.23 & 1.9 & 28 & 0.21 & 1.7 & 22 & 0.16 & 1.6 & 22 \\
\hline 5870.02 & $\mathrm{Fe}$ II & $44 \mathrm{~F}$ & 0.49 & 1.2 & 25 & 0.34 & 0.8 & 19 & 0.20 & 0.5 & 22 \\
\hline 5870.02 & $\mathrm{Fe} I I$ & $44 \mathrm{~F}$ & 0.12 & 1.9 & 25 & 0.09 & 1.7 & 19 & 0.16 & 1.6 & 22 \\
\hline 5875.615 & $\mathrm{He} \mathrm{I}$ & 11 & -10.9 & 4.9 & 34 & -4.63 & 3.8 & 36 & -4.97 & 2.8 & 15 \\
\hline 5889.953 & $\mathrm{Na} \mathrm{I}$ & & 3.67 & 3.2 & 48 & 4.57 & 3.5 & 5 & 2.52 & 3.0 & 6 \\
\hline 5889.953 & $\mathrm{Na} \mathrm{I}$ & & -1.33 & 1.2 & 31 & -1.76 & 0.6 & 22 & -2.41 & 0.5 & 22 \\
\hline 5895.923 & $\mathrm{Na} \mathrm{I}$ & & 3.14 & 3.2 & 48 & 4.41 & 3.5 & 4 & 2.79 & 2.3 & 9 \\
\hline 5895.923 & $\mathrm{Na} \mathrm{I}$ & & -0.96 & 1.2 & 31 & -1.65 & 0.6 & 24 & -2.56 & 0.5 & 24 \\
\hline 5955.802 & O II & & 1.26 & 5.3 & 61 & 0.79 & 1.8 & 21 & & & \\
\hline 5978.93 & Si II & 4 & 1.59 & 4.26 & 21 & 0.80 & 2.0 & 16 & 0.72 & 1.4 & 19 \\
\hline 6122.45 & Mn II & 13 & 0.53 & 1.2 & 28 & 0.78 & 1.2 & 23 & 0.72 & 1.2 & 19 \\
\hline 6122.45 & Mn II & 13 & 0.74 & 3.0 & 28 & 0.45 & 3.0 & 23 & 0.08 & 3.0 & 19 \\
\hline
\end{tabular}


Table 2. continued.

\begin{tabular}{|c|c|c|c|c|c|c|c|c|c|c|c|}
\hline$\overline{\text { IDENT }}$ & & & 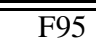 & "W95 & "V95 & $\overline{\text { F99 }}$ & 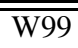 & "V99 & $\overline{\mathrm{F} \text { F01 }}$ & 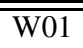 & $\overline{\mathrm{V} \text { V01 }}$ \\
\hline 6125.861 & Mn II & 13 & 0.36 & 1.2 & 25 & 0.51 & 1.2 & 25 & 0.56 & 1.2 & 22 \\
\hline 6125.861 & Mn II & 13 & 0.67 & 3.0 & 25 & 0.60 & 3.0 & 25 & 0.21 & 3.0 & 22 \\
\hline 6128.733 & Mn II & 13 & 0.30 & 1.2 & 28 & 0.39 & 1.2 & 28 & 0.43 & 1.2 & 26 \\
\hline 6128.733 & Mn II & 13 & 0.59 & 3.0 & 28 & 0.52 & 3.0 & 28 & 0.18 & 3.0 & 26 \\
\hline 6130.801 & Mn II & 13 & 0.24 & 1.2 & 39 & 0.27 & 1.2 & 38 & 0.40 & 1.2 & 36 \\
\hline 6130.801 & Mn II & 13 & 0.52 & 3.0 & 39 & 0.45 & 3.0 & 38 & 0.15 & 3.0 & 36 \\
\hline 6147.741 & $\mathrm{Fe}$ II & 74 & 0.07 & 1.3 & 18 & 0.21 & 1.3 & 23 & & & \\
\hline 6147.741 & $\mathrm{Fe} I I$ & 74 & 0.39 & 3.6 & 18 & 0.29 & 3.6 & 23 & & & \\
\hline 6147.741 & $\mathrm{Fe}$ II & 74 & -0.06 & 0.9 & 30 & -0.07 & 0.9 & 30 & & & \\
\hline 6149.258 & $\mathrm{Fe}$ II & 74 & 0.09 & 1.3 & 24 & 0.11 & 1.3 & 21 & & & \\
\hline 6149.258 & $\mathrm{Fe}$ II & 74 & 0.65 & 3.6 & 24 & 0.21 & 3.6 & 21 & & & \\
\hline 6149.258 & $\mathrm{Fe}$ II & 74 & -0.06 & 0.9 & 41 & -0.07 & 0.9 & 25 & & & \\
\hline 6158.019 & Fe II & 200 & 0.49 & 1.3 & 34 & 0.35 & 1.3 & 30 & 0.38 & 1.2 & 38 \\
\hline 6158.019 & $\mathrm{Fe}$ II & 200 & 0.51 & 3.6 & 34 & 0.13 & 3.6 & 30 & 0.26 & 3.6 & 38 \\
\hline 6158.019 & $\mathrm{Fe}$ II & 200 & -0.20 & 0.9 & 38 & -0.07 & 0.9 & 40 & & & \\
\hline 6160.755 & $\mathrm{Fe}$ II & 161 & 0.24 & 1.3 & 30 & 0.28 & 1.3 & 32 & & & \\
\hline 6160.755 & $\mathrm{Fe}$ II & 161 & 0.37 & 3.6 & 30 & 0.08 & 3.6 & 32 & & & \\
\hline 6160.755 & $\mathrm{Fe}$ II & 161 & -0.10 & 0.9 & 39 & -0.07 & 0.9 & 41 & & & \\
\hline 6161.086 & $\mathrm{Fe} I I$ & & & & & & & & 0.17 & 1.2 & 16 \\
\hline 6161.086 & Fe II & & & & & & & & 0.09 & 3.6 & 16 \\
\hline 6172.6 & $\mathrm{Fe}$ II & 98 & 0.24 & 1.3 & 29 & 0.29 & 1.3 & 29 & 0.18 & 1.2 & 24 \\
\hline 6172.6 & $\mathrm{Fe}$ II & 98 & 0.55 & 3.7 & 29 & 0.28 & 3.7 & 29 & 0.35 & 2.3 & 24 \\
\hline 6172.6 & $\mathrm{Fe}$ II & 98 & -0.11 & 1.0 & 32 & -0.07 & 0.8 & 37 & & & \\
\hline 6188.552 & $\mathrm{Fe}$ II & $44 \mathrm{~F}$ & 0.30 & 1.6 & 28 & 0.20 & 0.7 & 22 & 0.24 & 0.6 & 21 \\
\hline 6233.534 & $\mathrm{Fe} I I$ & 93 & 0.46 & 1.3 & 25 & 0.34 & 1.3 & 20 & 0.29 & 1.2 & 27 \\
\hline 6233.534 & $\mathrm{Fe}$ II & 93 & 0.88 & 3.7 & 25 & 0.24 & 3.7 & 20 & 0.29 & 3.0 & 27 \\
\hline 6233.534 & $\mathrm{Fe}$ II & 93 & -0.32 & 1.0 & 28 & -0.08 & 1.0 & 23 & -0.09 & 0.6 & 32 \\
\hline 6238.392 & $\mathrm{Fe} I I$ & 74 & 0.06 & 1.3 & 26 & 0.16 & 1.3 & 28 & & & \\
\hline 6238.392 & $\mathrm{Fe} I I$ & 74 & 0.43 & 3.6 & 26 & 0.21 & 3.7 & 28 & & & \\
\hline 6238.392 & $\mathrm{Fe} I I$ & 74 & -0.04 & 0.9 & 39 & -0.08 & 1.0 & 31 & & & \\
\hline 6239.953 & Fe II & 74 & 0.03 & 1.3 & 29 & 0.11 & 1.3 & 26 & & & \\
\hline 6239.953 & $\mathrm{Fe}$ II & 74 & 0.29 & 3.6 & 29 & 0.12 & 3.7 & 26 & & & \\
\hline 6239.953 & $\mathrm{Fe} I I$ & 74 & -0.04 & 0.9 & 35 & -0.08 & 1.0 & 31 & & & \\
\hline 6247.557 & $\mathrm{Fe} I \mathrm{II}$ & 74 & 0.32 & 1.3 & 21 & 0.52 & 1.3 & 22 & 0.40 & 1.3 & 21 \\
\hline 6247.557 & $\mathrm{Fe} I \mathrm{II}$ & 74 & 0.32 & 3.7 & 21 & 0.37 & 3.7 & 22 & 0.19 & 3.7 & 21 \\
\hline 6247.557 & $\mathrm{Fe}$ II & 74 & -0.17 & 0.9 & 23 & -0.27 & 0.9 & 25 & -0.18 & 0.5 & 22 \\
\hline 6248.898 & $\mathrm{Fe} I I$ & 92 & 0.90 & 1.3 & 24 & 0.84 & 1.3 & 27 & 0.92 & 1.3 & 24 \\
\hline 6248.898 & $\mathrm{Fe}$ II & 92 & 1.46 & 3.7 & 24 & 0.55 & 3.7 & 27 & 0.37 & 3.7 & 24 \\
\hline 6248.898 & $\mathrm{Fe}$ II & 92 & -0.41 & 0.9 & 27 & -0.25 & 0.9 & 32 & -0.10 & 0.5 & 26 \\
\hline 6300.23 & O I & $\mathrm{F}$ & 18.76 & 1.5 & 34 & 17.26 & 0.9 & 27 & 15.20 & 0.8 & 27 \\
\hline 6317.394 & $\mathrm{Fe}$ II & 200 & 0.38 & 1.3 & 25 & & & & 0.39 & 1.3 & 25 \\
\hline 6317.394 & $\mathrm{Fe} I \mathrm{II}$ & 200 & 0.46 & 3.7 & 25 & & & & 0.56 & 3.7 & 25 \\
\hline 6317.983 & $\mathrm{Fe} I \mathrm{II}$ & 92 & 0.79 & 1.3 & 25 & 1.89 & 1.3 & 27 & 1.57 & 1.3 & 25 \\
\hline 6317.983 & $\mathrm{Fe} I I$ & 92 & 0.64 & 3.7 & 25 & 2.65 & 3.7 & 27 & 1.67 & 3.7 & 25 \\
\hline 6318.717 & Mg I & 23 & -0.14 & 0.9 & 18 & -0.25 & 0.8 & 4 & -0.36 & 0.6 & 16 \\
\hline 6318.717 & Mg I & 23 & 0.79 & 1.3 & 16 & & & & 0.46 & 1.3 & 16 \\
\hline 6318.717 & Mg I & 23 & 2.13 & 3.7 & 16 & & & & 0.37 & 3.7 & 16 \\
\hline 6346.742 & Mg II & 16 & 1.48 & 3.7 & -10 & 1.50 & 3.0 & 0 & 1.21 & 3.0 & 0 \\
\hline 6346.742 & Mg II & 16 & 1.15 & 5.8 & -10 & 0.87 & 5.8 & 0 & 0.53 & 5.8 & 0 \\
\hline 6346.742 & Mg II & 16 & -0.60 & 1.9 & -22 & -0.54 & 1.2 & 0 & -0.36 & 1.2 & 2 \\
\hline 6363.88 & O I & $1 \mathrm{~F}$ & 6.95 & 1.7 & 28 & 5.92 & 1.0 & 18 & 4.76 & 0.8 & 17 \\
\hline 6369.462 & $\mathrm{Fe} I I$ & 40 & 0.19 & 1.3 & 23 & 0.33 & 1.3 & 24 & 0.17 & 1.3 & 24 \\
\hline 6369.462 & $\mathrm{Fe}$ II & 40 & 0.27 & 3.7 & 23 & 0.55 & 3.7 & 24 & 0.23 & 3.7 & 24 \\
\hline 6371.125 & $\mathrm{Fe}$ II & 93 & 0.08 & 1.3 & 23 & 0.08 & 1.3 & 24 & 0.09 & 1.3 & 24 \\
\hline 6371.125 & $\mathrm{Fe}$ II & 93 & 0.14 & 3.7 & 23 & 0.09 & 3.7 & 24 & 0.15 & 3.7 & 24 \\
\hline 6371.371 & Si II & 2 & 0.26 & 1.3 & 23 & 0.04 & 1.3 & 23 & 0.07 & 1.3 & 24 \\
\hline 6371.371 & Si II & 2 & 0.23 & 3.7 & 23 & 0.11 & 3.7 & 23 & 0.75 & 3.7 & 24 \\
\hline
\end{tabular}


G. Muratorio et al.: Analysis of the circumstellar environment of the B[e] star HD 45677 (FS Canis Majoris), Online Material p 11

Table 2. continued.

\begin{tabular}{|c|c|c|c|c|c|c|c|c|c|c|c|}
\hline$\overline{\text { IDENT }}$ & & & F95 & W95 & V95 & F99 & W99 & V99 & $\overline{\text { F01 }}$ & W01 & $\overline{\mathrm{V} 01}$ \\
\hline 6383.722 & Fe II & 92 & 0.95 & 1.3 & 27 & 1.38 & 1.2 & 27 & 1.24 & 1.3 & 22 \\
\hline 6383.722 & $\mathrm{Fe}$ II & 92 & 1.67 & 3.6 & 27 & 0.92 & 3.8 & 27 & 1.64 & 3.7 & 22 \\
\hline 6383.722 & Fe II & 92 & -0.22 & 0.9 & 30 & -0.23 & 0.8 & 31 & & & \\
\hline 6385.451 & $\mathrm{Fe}$ II & 92 & 0.61 & 1.3 & 28 & 0.92 & 1.2 & 23 & 0.85 & 1.3 & 23 \\
\hline 6385.451 & $\mathrm{Fe}$ II & 92 & 1.73 & 3.6 & 28 & 0.62 & 3.8 & 23 & 0.82 & 3.7 & 23 \\
\hline 6385.451 & $\mathrm{Fe}$ II & 92 & -0.22 & 0.9 & 32 & -0.16 & 0.8 & 28 & & & \\
\hline 6416.919 & $\mathrm{Fe}$ II & 74 & 0.16 & 1.3 & 33 & 0.32 & 1.3 & 28 & 0.47 & 1.3 & 25 \\
\hline 6416.919 & $\mathrm{Fe}$ II & 74 & 0.74 & 3.7 & 33 & 0.18 & 3.7 & 28 & 0.19 & 3.7 & 25 \\
\hline 6416.919 & $\mathrm{Fe}$ II & 74 & -0.03 & 1.0 & 53 & -0.06 & 0.8 & 26 & -0.15 & 0.6 & 26 \\
\hline 6440.4 & $\mathrm{Fe} \mathrm{II}$ & $15 \mathrm{~F}$ & 0.19 & 1.3 & 32 & 0.27 & 1.1 & 26 & 0.17 & 0.7 & 24 \\
\hline 6442.955 & $\mathrm{Fe}$ II & 93 & 0.43 & 1.3 & 30 & 0.53 & 1.2 & 24 & 0.71 & 1.3 & 22 \\
\hline 6442.955 & $\mathrm{Fe} \mathrm{II}$ & 93 & 1.24 & 3.7 & 30 & 0.67 & 3.7 & 24 & 0.60 & 3.7 & 22 \\
\hline 6442.955 & $\mathrm{Fe}$ II & 93 & -0.10 & 1.0 & 36 & -0.11 & 1.0 & 30 & -0.06 & 0.6 & 28 \\
\hline 6455.837 & $\mathrm{Fe}$ II & 92 & 0.15 & 1.3 & 29 & 0.56 & 1.3 & 29 & 0.39 & 1.3 & 25 \\
\hline 6455.837 & $\mathrm{Fe}$ II & 92 & 0.20 & 3.7 & 29 & 0.28 & 3.7 & 29 & 0.37 & 3.7 & 25 \\
\hline 6455.837 & $\mathrm{Fe}$ II & 92 & -0.27 & 1.0 & 34 & -0.10 & 0.9 & 33 & -0.04 & 0.5 & 29 \\
\hline 6456.383 & $\mathrm{Fe}$ II & 74 & 1.12 & 1.3 & 28 & 1.13 & 1.3 & 29 & 1.18 & 1.3 & 22 \\
\hline 6456.383 & $\mathrm{Fe}$ II & 74 & 1.93 & 3.7 & 28 & 0.40 & 3.7 & 29 & 0.75 & 3.7 & 22 \\
\hline 6456.383 & $\mathrm{Fe} \mathrm{II}$ & 74 & -0.27 & 1.0 & 34 & -0.39 & 0.9 & 32 & -0.34 & 0.5 & 23 \\
\hline 6482.204 & $\mathrm{Fe}$ II & 199 & 0.30 & 1.3 & 28 & 0.41 & 1.3 & -3 & & & \\
\hline 6482.204 & $\mathrm{Fe}$ II & 199 & 0.52 & 3.7 & 28 & 0.56 & 3.6 & -3 & & & \\
\hline 6482.204 & $\mathrm{Fe}$ II & 199 & -0.11 & 1.0 & 30 & -0.12 & 1.0 & -3 & & & \\
\hline 6485.282 & $\mathrm{Fe}$ II & $90 \mathrm{~F}$ & 0.55 & 1.6 & 18 & 0.46 & 1.1 & 16 & & & \\
\hline 6491.246 & $\mathrm{Fe}$ II & 92 & 0.33 & 1.3 & 30 & 0.70 & 1.2 & 22 & 0.79 & 1.3 & 24 \\
\hline 6491.246 & $\mathrm{Fe}$ II & 92 & 0.64 & 3.7 & 30 & 0.27 & 3.8 & 22 & 0.37 & 3.7 & 24 \\
\hline 6491.246 & $\mathrm{Fe}$ II & 92 & -0.06 & 1.0 & 33 & -0.19 & 0.8 & 29 & -0.10 & 0.5 & 27 \\
\hline 6493.035 & $\mathrm{Fe} \mathrm{II}$ & 92 & 0.37 & 1.3 & 28 & 0.83 & 1.2 & 28 & 0.72 & 1.3 & 27 \\
\hline 6493.035 & $\mathrm{Fe}$ II & 92 & 1.32 & 3.7 & 28 & 0.27 & 3.8 & 28 & 0.37 & 3.7 & 27 \\
\hline 6493.035 & $\mathrm{Fe}$ II & 92 & -0.06 & 1.0 & 33 & -0.19 & 0.8 & 36 & -0.05 & 0.5 & 30 \\
\hline 6506.333 & $\mathrm{Fe} \mathrm{II}$ & 93 & 0.40 & 1.9 & 29 & 0.46 & 1.3 & 24 & 0.37 & 1.3 & 22 \\
\hline 6506.333 & $\mathrm{Fe}$ II & 93 & 0.31 & 3.7 & 29 & 0.22 & 3.7 & 24 & 0.08 & 3.7 & 22 \\
\hline 6506.333 & $\mathrm{Fe}$ II & 93 & -0.06 & 1.0 & 29 & -0.20 & 0.8 & 28 & -0.06 & 0.6 & 24 \\
\hline 6517.018 & $\mathrm{Fe}$ II & 92 & 0.63 & 1.3 & 29 & 0.92 & 1.3 & 27 & 0.66 & 1.3 & 21 \\
\hline 6517.018 & $\mathrm{Fe} \mathrm{II}$ & 92 & 0.86 & 3.7 & 28 & 0.45 & 3.7 & 27 & 0.22 & 3.7 & 21 \\
\hline 6517.018 & $\mathrm{Fe} \mathrm{II}$ & 92 & & & & -0.20 & 0.7 & 31 & -0.06 & 0.6 & 25 \\
\hline
\end{tabular}


Table 3. Atomic species identified in our spectra of HD 45677. For each Moore's Table multiplet, the number of emission and absorption lines is indicated.

\begin{tabular}{|c|c|}
\hline Species & Multiplets \\
\hline $\mathrm{Ca} \mathrm{I}$ & $23(1 \mathrm{e}, 1 \mathrm{a})$ \\
\hline Cr II & $26(1 \mathrm{e}, 1 \mathrm{a}) ; 30(2 \mathrm{e}, 2 \mathrm{a}) ; 31(1 \mathrm{e}, 1 \mathrm{a}) ; 43(1 \mathrm{e}, 1 \mathrm{a}) ; 44(6 \mathrm{e}, 6 \mathrm{a})$ \\
\hline$[\mathrm{Cu} \mathrm{II}]$ & $1(1)$ \\
\hline $\mathrm{Fe}$ II & $\begin{array}{l}\text { 22(1e);27(7e,7a);28(2e,2a);32(3e,1a);37(9e, ,9a); } \\
\text { 38(7e,7a);40(1e);41(1e,1a);42(3e,3a);48(2e,2a); } \\
\text { 49(7e,7a);55(1e,1a);74(7e,7a);(92(8e,7a));(93(4e,3a)); } \\
(97(1 \mathrm{e})) ;(98(1 \mathrm{e}, 1 \mathrm{a})) ; 161(1 \mathrm{e}, 1 \mathrm{a}) ; 199(1 \mathrm{e}, 1 \mathrm{a}) ; 200(2 \mathrm{e}, 1 \mathrm{a})\end{array}$ \\
\hline$[\mathrm{Fe} \mathrm{II}]$ & $\begin{array}{l}4(7) ; 6(10) ; 7(5) ; 15(1) ; 17(5) ; 18(8) ; 19(8) ; 20(10) ; 21(11) ; \\
23(2) ; 34(2) ; 35(4) ; 39(5) ; 44(3) ; 90(1) ; 97(2) ; 98(1)\end{array}$ \\
\hline $\mathrm{H}$ & $\mathrm{H}_{\delta} \mathrm{H}_{\gamma} \mathrm{H}_{\beta}$ \\
\hline $\mathrm{He}$ & $\begin{array}{l}\text { 4(1a);11(1a);12(1a);14(1a);16(1a);47(1a); } \\
\text { 48(1a);50(1a);51(1a);53(1a) }\end{array}$ \\
\hline Mg I & $1(1 \mathrm{e}, 1 \mathrm{a}) ; 2(3 \mathrm{e}, 1 \mathrm{a}) ; 23(1 \mathrm{e}, 1 \mathrm{a})$ \\
\hline $\mathrm{Mg}$ II & $4(1 \mathrm{e}, 1 \mathrm{a}) ; 16(1 \mathrm{e}, 1 \mathrm{a})$ \\
\hline Mn II & $13(4 \mathrm{e})$ \\
\hline$[\mathrm{N} \mathrm{II}]$ & $3(1)$ \\
\hline $\mathrm{NaI}$ & $1(2 \mathrm{e}, 2 \mathrm{a})$ \\
\hline [Ni II] & $3(3)$ \\
\hline$[\mathrm{O} I]$ & $1(2)$ \\
\hline$[\mathrm{S}$ II $]$ & $1(2)$ \\
\hline Si II & $2(1 \mathrm{e}) ; 3(2 \mathrm{a}) ; 4(1 \mathrm{e})$ \\
\hline Ti II & $38(1 \mathrm{e}) ; 39(1 \mathrm{e}) ; 82(1 \mathrm{e})$ \\
\hline
\end{tabular}


G. Muratorio et al.: Analysis of the circumstellar environment of the B[e] star HD 45677 (FS Canis Majoris), Online Material p 13
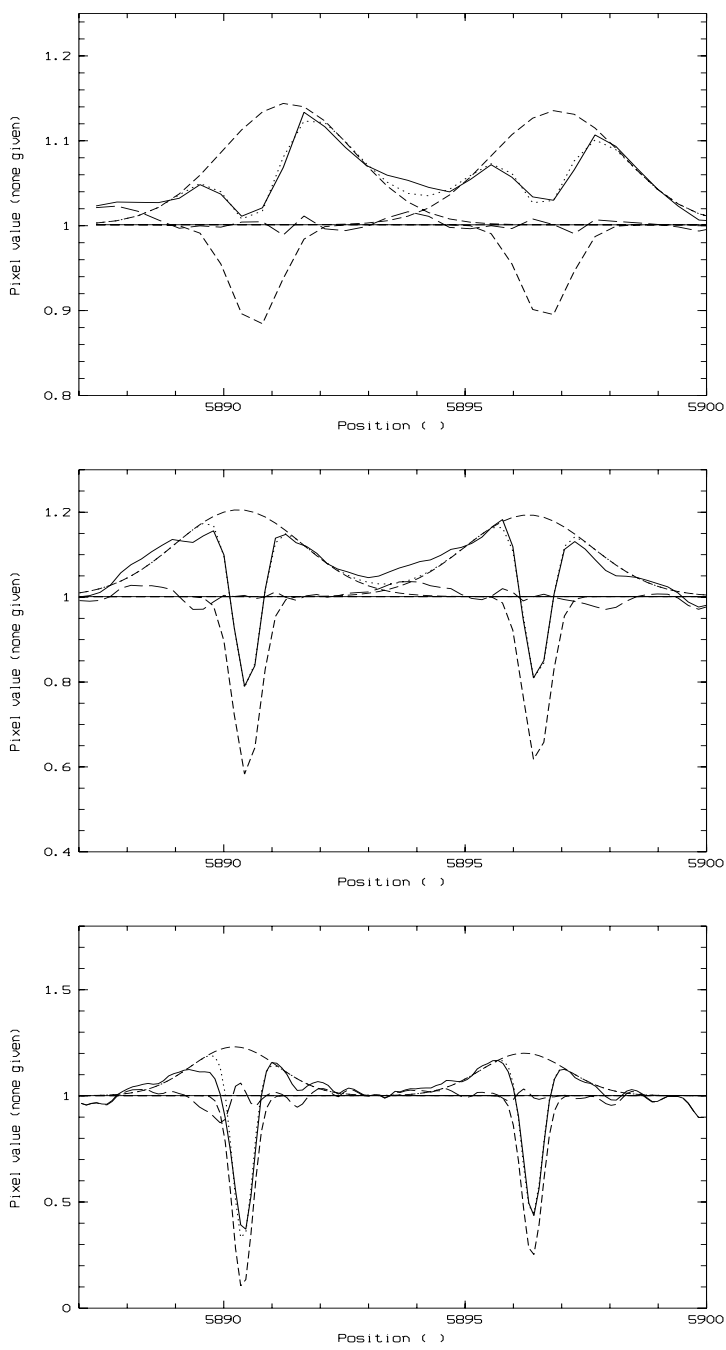

Fig. 3. The Na ID lines in 1995 (top), 1999 (middle), 2002 (bottom) and the fitted components. The profile (solid line) is fitted (dotted line) by two Gaussian components whose parameters (wavelength, flux, $F W H M$ ) are displayed in the right upper corner. The residual (long dashed line) appears around the continuum (straight line). 